\title{
UNIFIED ELECTRON-ION RECOMBINATION RATE COEFFICIENTS OF SILICON AND SULFUR IONS
}

\author{
Sultana N. Nahar and Anil K. Pradhan \\ Department of Astronomy, The Ohio State University, Columbus, OH 43210 \\ Received 1994 September 12; accepted 1994 December 15
}

\begin{abstract}
Total recombination rate coefficients for the astrophysically important $\mathrm{Si}$ and $\mathrm{S}$ ions, $\mathrm{Si}$ I, $\mathrm{Si}$ I, Si $\mathrm{Ix}, \mathrm{S} \mathrm{n}$, and $\mathbf{S}$ III, are obtained employing a new unified treatment developed for electron-ion recombination. The treatment incorporates both the radiative and the dielectronic recombination processes in the close coupling approximation from atomic collision theory, and the calculations are carried out using the $R$-matrix method as developed for the Opacity Project. All recombined states from the ground state to $n=\infty$ are considered. The states are divided into two groups, a low- $n$ and a high-n group. Detailed partial photoionization cross sections into the ground state of the recombining ion, including autoionizing resonances due to coupling to excited states of the ion, are calculated for all bound states in the low-n group, and the Milne relation is used to calculate the corresponding contribution to the recombination rate coefficient. For the high-n group, collision strengths for dielectronic recombination, both detailed and resonance averaged, are obtained using the precise theory of Bell \& Seaton. Total recombination rate coefficients are computed over a wide range of temperatures for practical purposes. A discussion of the atomic effects that determine the accuracy of the recombination rates is presented, in particular as a function of the increase in the eigenfunction expansion for the recombining ion, studied for $e+\mathrm{Si} \mathrm{x} \rightarrow \mathrm{Si}$ IX. Comparison is made with earlier works.
\end{abstract}

Subject headings: atomic data - atomic processes

\section{INTRODUCTION}

Determination of the ionization structure of astrophysical and laboratory plasmas requires the knowledge of recombination rates of electrons with atomic ions. For example, the determination of abundances from observed spectral features depends on the ionization fractions of elements which, in turn, are obtained by relating the rates of ionization and recombination. In general the relevant rates are needed for all ionization stages of all astrophysically abundant elements. However, particular elements often characterize or dominate the observed spectra of certain sources. One such example is the interstellar medium (ISM) where a few elements in low ionization stages are prime constituents (Spitzer \& Fitzpatrick 1993). Silicon and sulfur ions are present in the ionized gas in the ISM, and recombination rates are required for the analysis of abundances, grain depletion, and overall structure of the ISM. Some of these ions (e.g., S II) also enable useful density and temperature diagnostics of nebular and AGN plasmas (Osterbrock 1989). The present study employs a newly developed method to obtain accurate, total electron-ion recombination cross sections and rate coefficients. Important details of the method are outlined, and astrophysical implications are pointed out.

Assuming that the initial population in the excited states of ions is negligible, electron-ion recombination may take place to an infinite number of bound states of the recombined ion and through infinite series of autoionizing states in the continuum above the ground state of the recombining ion. Electronion recombination is generally, but artificially, divided into two separate processes (Osterbrock 1989): radiative recombination (RR), inverse of direct photoionization,

$$
\mathrm{X}^{+}+h v \rightarrow \mathrm{X}^{++}+\epsilon_{l},
$$

and dielectronic recombination (DR),

$$
e+\mathrm{X}^{++} \rightarrow\left(\mathrm{X}^{+}\right)^{* *} \rightarrow\left\{\begin{array}{l}
e+\mathrm{X}^{++} \\
\mathrm{X}^{+}+h v
\end{array} .\right.
$$

DR is a two-step process where the incident electron combines with the target ion in a doubly excited autoionizing state, which can lead to either autoionization or to a pure bound state through radiative decay of the excited core ion. Previous calculations of recombination rates have been carried out separately for the two processes RR and DR, the former by calculating photoionization cross sections neglecting resonances (e.g., Aldrovandi \& Pequignot 1973), and the latter by considering the resonances alone, usually in the so-called isolated resonance approximation (e.g., Badnell 1991). The RR is usually regarded to be important at low temperatures, whereas the DR, which requires electrons energetic enough to excite the ion, comes in at higher temperatures. However, even at low electron energies (temperatures), there may occur significant recombination of electrons through low-lying resonances near the ionization threshold (Nussbaumer \& Storey 1983). Thus in order to obtain total electron-ion recombination rates one needs to consider three processes: simple RR, high- $T$ DR, and low- $T$ DR, that have heretofore been treated individually and in different approximations.

In earlier works (Nahar \& Pradhan 1992a, 1992b, 1994; hereafter NP1, NP2, NP3) we have developed a unified treatment of electron-ion recombination that subsumes the RR and the DR processes in an ab initio manner within the close coupling (CC) approximation of atomic collision theory. The CC method has been widely employed for electron-ion scattering and photoionization calculations. The extension to electronion recombination entails a combination of the types of calculations, carried out using the $R$-matrix method (Berrington et 
al. 1987) and applied to large-scale calculations of photoionization cross sections, as in the Opacity Project (Seaton 1987).

The basic steps of the new method correspond to the physical processes illustrated in Figure 1. The present calculations pertain to the assumption that all recombining ions are initially in the ground state, i.e., the low-density approximation in astrophysical plasmas where excited states have negligible population. We divide the infinity of bound states of the final, recombined ion two groups: group $A$, low- $n$ states, $n \leq n_{0}$; and group B, high- $n$ states, $n_{0}<n \leq \infty$. Group A states, with $n_{0} \sim 10$, require detailed photoionization calculations for all such bound states at a large number of photon energies to delineate the extensive resonance structures in the partial photoionization cross sections leaving the recombining ion in the ground state. Detailed balance considerations, i.e., the Milne relation (Osterbrock 1989), yield the contribution to recombination corresponding to group $A$ states. The $a b$ initio calculation of resonance profiles in the photoionization cross sections of all possible $(e+$ ion) bound states of group $A$ ensures that both the RR and DR processes are accounted for. However, this needs to be complemented by recombinations to the high-n states, which predominantly takes place through autoionizing resonances, with $n_{0}<n \leq \infty$, covering onto the excited states of the recombining ion. The resonances decay radiatively via core transitions to the ground state in the recombining ion, with the outer high- $n$ electron essentially remaining a "spectator"; i.e., the DR process. In the energy region corresponding to these high- $n$ states, recombination through the "background" (nonresonant) part is very small and may be neglected (although it is included in the present work in the hydrogenic approximation). We employ the theory of DR developed by Bell \& Seaton (1985) using close coupled wavefunctions for the electron-ion system. The Bell and Seaton theory properly accounts for the electron-electron and electron-photon interactions and leads to a generalized $S$ matrix which satisfies the unitarity condition for the electron and photon fluxes, i.e., the sum of the scattering and recombining electrons is conserved; the latter is equal to the number of photons emitted. The DR calculations are carried out, using the $\boldsymbol{R}$-matrix method, as an extension of electron-ion scattering calculations.

Once the total electron-ion recombination rate coefficient is known, the equations of ionization balance may be written as

$$
N_{\mathrm{X}}+\int_{v_{0}}^{\infty} \frac{4 \pi J_{\mathrm{v}}}{h v} \sigma_{\mathrm{P}}(v) d v=N_{\mathrm{e}} N_{\mathrm{X}++} \alpha_{\mathrm{R}}\left(\mathrm{X}^{+} ; T\right),
$$

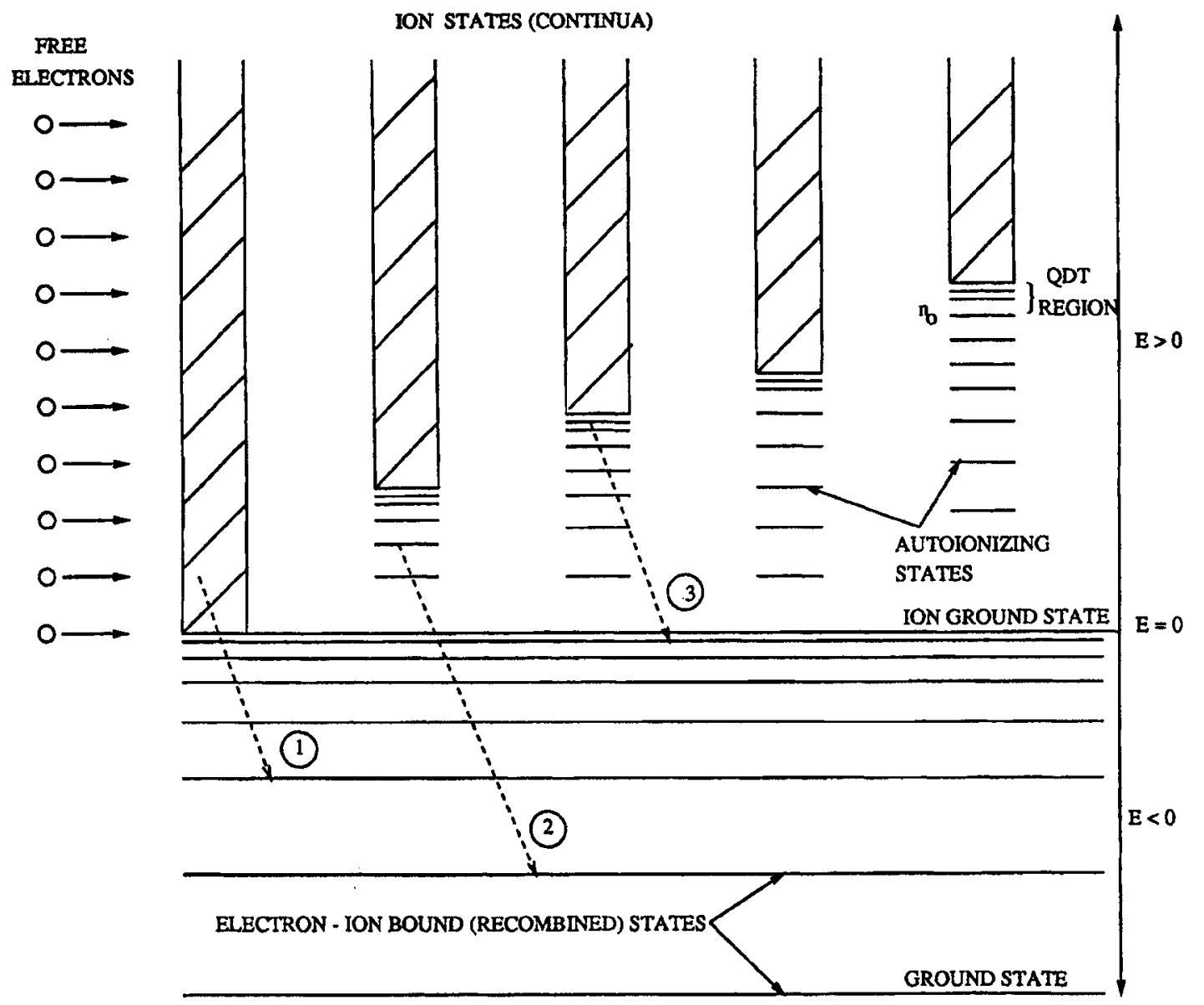

Fig. 1. - Schematic energy diagram for electron-ion recombination. The infinite series of autoionizing resonances converging onto the various excited target states are in the positive energy region $E>0$, while recombined states are in the negative energy region $E<0$. Broken lines with arrows represent photon emission during recombination: (1) recombination through the ground state continuum of the recombining ion, (2) through a low-n autoionizing state with possibly large interaction with the continua (coupling to excited states gives rise to autoionizing resonances), and (3) through a high-n autoionizing state, with negligible continuum contribution (DR only). 
in photoionization equilibrium, and

$$
C_{l} N_{e} N_{\mathrm{X}^{+}}=N_{e} N_{\mathrm{X}^{++}} \alpha_{\mathrm{R}}\left(\mathrm{X}^{+} ; T\right) \text {, }
$$

in coronal equilibrium, where $\sigma_{\mathrm{P} 1}$ is the total ground state photoionization cross section, $C_{\mathrm{I}}$ is the collisional ionization rate coefficient, and $J_{y}$ is the radiation field intensity. $\alpha_{R}$ is now the total recombination rate coefficient that substitutes the sum of RR and DR rates usually considered (Osterbrock 1989).

The present report also discusses some of the atomic effects that determine the accuracy of the calculations, in particular the convergence of the recombination cross sections as a function of the number of target ion states included in the CC calculations. In earlier works, we have computed $\alpha_{R}(T)$ for ions in the carbon isoelectronic sequence, and a few other ions such as C I (NP1, NP2, 1992; NP3, 1994). In this study the following ions are considered:

$$
\begin{aligned}
& e+\text { Si II } \rightarrow \text { Si I }, \\
& e+\text { Si III } \rightarrow \text { Si II, } \\
& e+\text { Si } x \rightarrow \text { Si Ix, } \\
& e+\text { S III } \rightarrow \text { S II }, \\
& e+\text { S IV } \rightarrow \text { S III } .
\end{aligned}
$$

\section{THEORY AND COMPUTATIONS}

The two main components of the present recombination calculations are as follows: group A, photoionization cross sections for all low- $n$ bound states of the recombined ion and group B, collision strengths for DR with high- $n$ autoionizing resonances that undergo radiative decay via dipole transitions between states of the recombining core ion. These are discussed below with selected results for the ions considered.

\subsection{Photoionization}

The partial photoionization cross sections, $\sigma_{\mathrm{pl}}(g)$, into the ground state of the recombining ion are calculated for all bound states of group A including detailed energy resolution of several infinite series of autoionizing resonances converging on to the various excited states of the core or target ion included in the close coupling expansion. The target states, $S_{1} L_{t} \pi_{t}$, included in the CC eigenfunction expansion for each ion considered are listed in Table 1. The table also lists the total number of group A bound LS terms, $N_{\text {bnd }}$, corresponding to $S L \pi=S_{1} L_{t} \pi_{i} n l$, with $n \leq n_{0}=10$ and $l \leq l_{\max }=9$. Bound states that do not couple to the target ground state $S_{i}$, i.e., with $\left|S_{i}^{\dagger}-S\right|>1$, such as the ${ }^{5} S^{\circ}$ state of Si I or $S \mathrm{II}$, are excluded from $N_{\text {bnd }}$ (these states may be significant contributors in dense plasmas).

The partial cross sections, $\sigma_{\mathrm{Rc}}\left(g, i_{b}\right)$, for the recombination to the bound state $i_{b}$ can be obtained from the photoionization cross sections using the Milne relation

$$
\sigma_{\mathrm{RC}}\left(g, i_{b}\right)=\frac{g_{i}}{g_{j}} \frac{h^{2} v^{2}}{m^{2} c^{2} v^{2}} \sigma_{\mathrm{PI}}\left(g, i_{b}\right),
$$

where $g_{i}$ and $g_{j}$ are the statistical weight factors of the bound $(e+i o n)$ state $i_{b}$ and of the residual ion ground state $(g)$,

\begin{tabular}{|c|c|c|c|c|c|}
\hline \multirow{2}{*}{ 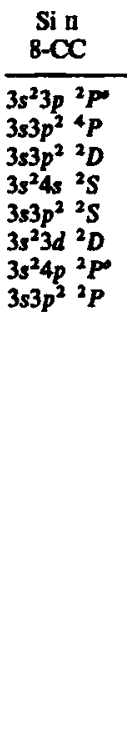 } & $\underset{12-C C}{\text { Si m }}$ & \multicolumn{2}{|c|}{$\underset{\text { 23-CC }}{\text { Si } x}$} & $\underset{17-C C}{S \text { mII }}$ & $\underset{16-C C}{S ~ I V ~}$ \\
\hline & 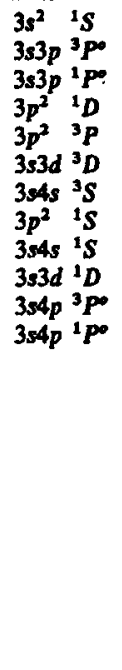 & $\begin{array}{l}2 s^{3} 2 p \\
2 s 2 p^{2} \\
2 s 2 p^{2} \\
2 s 2 p^{2} \\
2 s 2 p^{2} \\
2 p^{3} \\
2 p^{3} \\
2 p^{3} \\
2 s^{2} 3 s \\
2 s^{2} 3 p \\
2 s^{2} 3 d \\
2 s 2 p^{3} P \\
2 s 2 p^{3} P \\
2 s 2 p^{3} P \\
2 s 2 p^{3} P \\
2 s 2 p^{3} P \\
2 s 2 p^{3} P \\
2 s 2 p^{3} p \\
2 s 2 p^{3} P \\
2 s 2 p^{3} P \\
2 s 2 p^{1} P \\
2 s 2 p^{1} P \\
2 s 2 p^{1} P\end{array}$ & 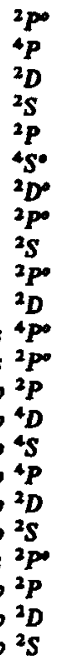 & 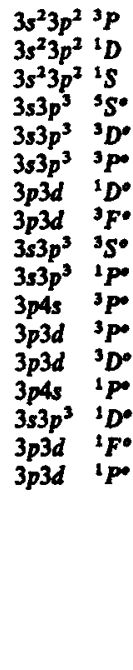 & 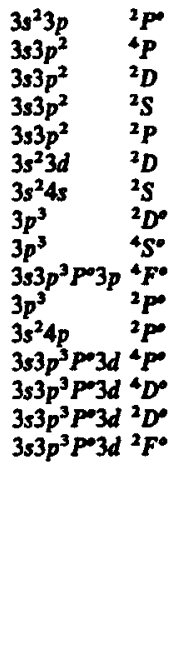 \\
\hline$N_{\text {bad }}=$ & 59 & & & 313 & 298 \\
\hline
\end{tabular}
respectively; $v$ is the velocity of the photoelectron; and $v$ is the photon frequency. The partial recombination rate coeficient, $\alpha_{R}$, for the recombined state $i_{b}$ can be obtained through averag-
TABLE 1

TARGBT STATES, $S_{i} L_{4} \pi_{t}$, INCLUDED IN THE WAVBIUNCTON EXPANSION POR TFI RECOMGINING IONS

Note- $-N_{\text {bod }}$ corresponds to total number of bound states formed from $S_{1} L_{t} \pi, n l$, with $n \leq 10$ (=11 in a few cases), $I \leq l_{\max }$ where $L_{\max }=8$ for Si $u_{1}$ $I_{1}=9$ for Si II, Si $x, S$ m, and S jv. Photoionization cross soctions are obtained and are included in the total recombination rate coefficients for all $N_{\text {bod }}$ bound states.

ing over the recombination cross sections as

$$
\alpha_{R}\left(i_{b}, T\right)=\int_{0}^{\infty} v f(v) \sigma_{\mathrm{R}}\left(g, i_{b}, v\right) d v,
$$

where $f(v)$ is the distribution function. Assuming Maxwellian distribution of electrons, the contribution from the low- $n$ states to the total recombination rate coefficient is

$$
\begin{aligned}
\alpha_{R}\left(T ; n \leq n_{0}\right) \\
\quad=\sum_{b_{b}}^{N_{b a d}} \frac{g_{i}}{g_{j}} \frac{2}{k T \sqrt{2 \pi m^{3} k T c^{2}}} \int_{0}^{\infty} E^{2} \sigma_{\mathrm{pr}}\left(g, i_{b} ; \epsilon\right) e^{-\epsilon / k T} d \epsilon,
\end{aligned}
$$

where $E=h v=\epsilon+I_{p}, \epsilon$ is the photoelectron energy, $I_{p}$ is the ionization potential, and the sum is over partial recombination rates of the bound states $i_{b}$ extending over the total number of bound states $N_{\text {bnd. }}$. Although large number of bound states are considered for each ion, a relatively small number dominates the total recombination rate coefficient at low and intermediate temperatures (the number of dominant states usually increases with temperature).

The low- $n \alpha_{R}\left(T ; n \leq n_{0}\right)$ calculated using the partial photoionization cross sections $\sigma_{\mathrm{Pr}}\left(g, i_{b}\right)$ closely approximate those calculated with the total cross sections $\sigma_{\mathrm{pt}}\left(i_{b}\right)$ provided the first excited core state lies significantly above the ground state, such as for the cross sections considered in NP3 [where they employed $\sigma_{\mathrm{P}(}\left(i_{b}\right)$ instead of $\left.\sigma_{\mathrm{P}}\left(g, i_{b}\right)\right]$. This is because (1) $\sigma_{\mathrm{p}}\left(i_{b}\right)=\sigma_{\mathrm{pr}}\left(g, i_{b}\right)$ up to the first excited state of the recombining ion, often a few eV above the ground state; (2) the partial 
photoionization cross sections into the excited states, are much smaller than those into the ground state (e.g., Fig. 2, and Nahar \& Pradhan 1992c, 1993); (3) the exponential term in equation (3) involves the energy difference relative to the ground state and therefore the excited state contributions are exponentially damped out; and (4) the high- $n$ recombination (DR) dominates above $T>10^{4} \mathrm{~K}$, at electron energies more than a few $\mathrm{eV}$. Figure 2 presents the total and the partial photoionization cross sections for the ground state $3 s^{2} 3 p^{2}\left({ }^{3} P\right)$ of S III; Figure $2 a$ shows the total $\sigma_{\mathrm{PI}}\left({ }^{3} P\right)$, whereas Figures $2 b$ and $2 c$ show the partial cross sections; $\sigma_{\mathrm{pr}}\left({ }^{3} P \rightarrow{ }^{2} P^{\circ}\right)$ into the ground state $\left.3 s^{2} 3 p^{2} P^{0}\right)$, and $\sigma_{\mathrm{P}(}\left({ }^{3} P \rightarrow{ }^{4} P\right)$ into the $3 s 3 p^{2}\left({ }^{4} P\right)$ state of the target ion $S \mathrm{IV}$, respectively. The figure shows that the partial $\sigma_{\mathrm{Pf}}\left({ }^{3} P \rightarrow{ }^{2} P^{\circ}\right)$ very closely approximates the total $\sigma_{\mathrm{Pr}}\left({ }^{3} P\right)$. For the ions considered in NP3, the first excited state lies $>5 \mathrm{eV}$ above the ground state; consequently the rate coefficients calculated using $\sigma_{\mathrm{Pl}}$ differ little from those calculated using $\sigma_{\mathrm{pr}}\left(g, i_{b}\right)$ by less than $10 \%$. There are some additional differences, though, approximately $10 \%-20 \%$, due to the inclusion in NP3 of $\left(e+\right.$ ion) symmetries with $\left|S_{t}-S\right|>1$ not coupled to the ground state of the ion. In general for low-density plasmas the $\sigma_{\mathrm{PI}}\left(g, i_{b}\right)$ should be employed in the calculations of the low- $n$ rate coefficients, in exact conformity with the Milne

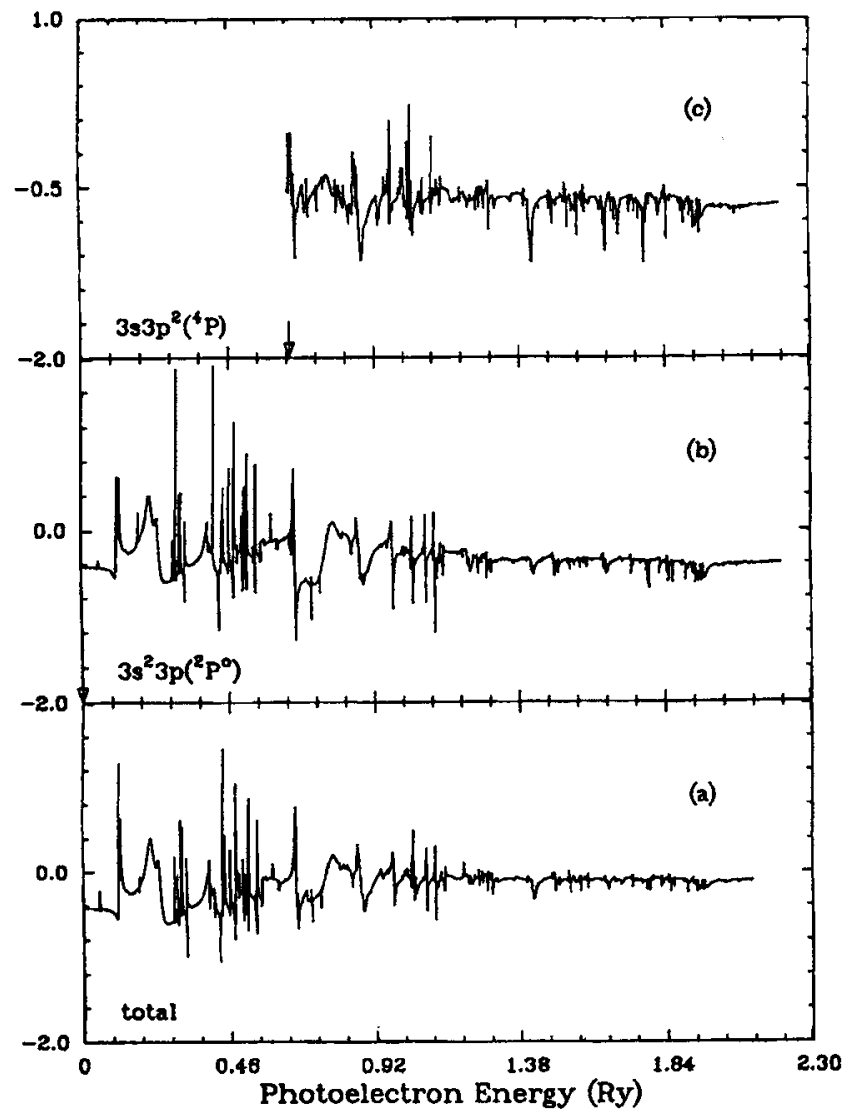

Fio. 2-Total and partial cross sections for photoionization of the ground state $3 s^{2} 3 p^{2}\left({ }^{3} P\right)$ of $\mathrm{S} \mathrm{m}$, (a) the total cross section $\sigma_{\mathrm{m}}\left({ }^{3} P\right)$ includes the sum over partial cross sections into the ground and excited states of $S$ iv (see Table 1), (b) the partial cross section $\sigma_{\mathrm{m}}\left(\mathrm{g}^{3},{ }^{3} P \rightarrow{ }^{2} P^{\circ}\right)$ into the ground state of $S \mathrm{IV}$, and $(c)$ partial cross section $\sigma_{\mathrm{Pf}}\left({ }^{3} P \rightarrow{ }^{4} P\right)$ into the first excited state ${ }^{4} P$. Arrows in the upper two panels indicate the energy position of the target thresholds. Except for the top panel, the upper limit of the $y$-axis is not shown. relation (the case of recombination through excited states of the recombining ion will be discussed in subsequent works).

Prior to the Opacity Project (OP), photoionization cross sections for excited bound states of the recombined $(e+$ ion) system were usually considered to be quasi-hydrogenic, with the assumption that the excited electron is far enough from the core ion where the electron-electron interaction can be neglected. This assumption is not valid for complex atoms, especially when the core electrons may be photoexcited and thus enhance the photoionization cross sections. The enhancement can be up to several orders of magnitude relative to the background photoionization and manifests itself through resonances that are very much wider than the narrow Rydberg autoionizing resonances. Figure 3 presents the partial photoionization cross sections of eight excited bound states in a Rydberg series, $3 s^{2} 3 p n s{ }^{3} p^{o}, 4 \leq n \leq 11$, of $S$ III into the ground state of ${ }^{2} P^{\circ}$ of $S$ IV; the arrows point to the energies of the dipole-allowed transitions for the $S$ IV core (see Table 2). At energies of these states, the core is photoexcited while the outer electron remains basically a "spectator" (as in the DR process), a phenomenon known as the PEC (photoexcitationof-core) resonance (Yu \& Seaton 1987; Nahar \& Pradhan 1993). The PEC resonances become more prominent as " $n$ "

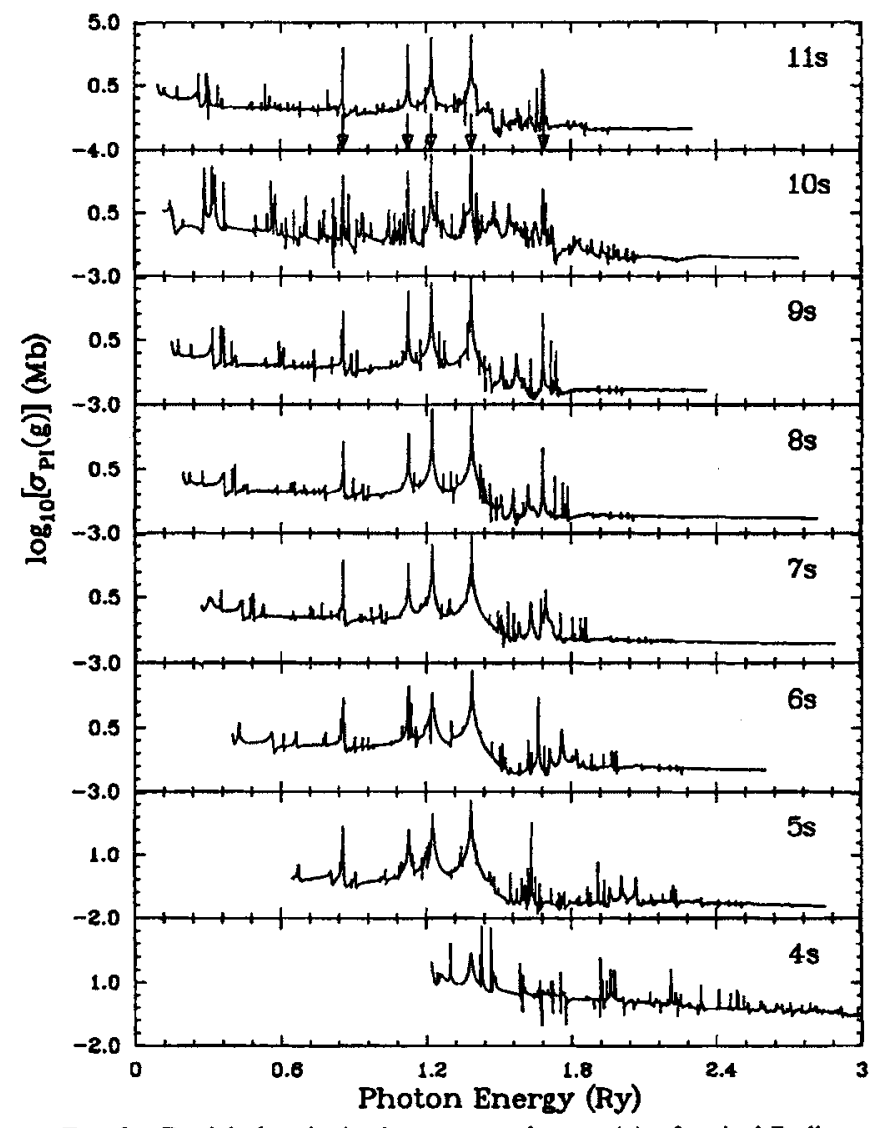

Fig. 3.-Partial photoionization cross sections, $\sigma_{\mathrm{p}}(g)$, of excited Rydberg series of bound states, $3 s^{2} 3 p m s{ }^{3} P^{\circ}, 4 \leq n \leq 11$, of $\mathrm{S} n$ into the ground state ${ }^{2} P^{0}$ of $S \mathrm{IV}$. Except for the top panel, the upper limits of the cross sections are not shown in the panels. The arrows point to the energies of excited thresholds $3 s 3 p^{2}\left({ }^{2} D,{ }^{2} S,{ }^{2} P\right), 3 s^{2} 3 d^{2} D$ and $3 s^{2} 4 s^{2} S$ of the core ion, $S$ IV, where PEC occurs. The PEC resonances appear as wide resonances. The PECs are prominent features in excited state photoionization cross sections. 
TABLE 2

Dipole Allowed Transitions from the Ground to Excited States of the Rbcomminano lons

\begin{tabular}{|c|c|c|c|}
\hline \multirow[b]{2}{*}{ Recombining ION } & \multirow[b]{2}{*}{ Ground State } & \multicolumn{2}{|r|}{ Exciteo States } \\
\hline & & $\Delta n=0$ & $\Delta n \neq 0$ \\
\hline 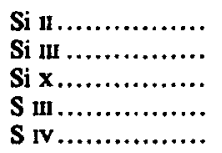 & $\begin{array}{ll}3 s^{2} 3 p & 2 p \\
3 s^{2} & { }^{1} S \\
2 s^{2} 2 p & { }^{2} p \\
3 s^{2} 3 p^{2} & { }^{3} p \\
3 s^{2} 3 p & { }^{2} p^{0}\end{array}$ & $\begin{array}{l}3 s 3 p^{2}\left({ }^{2} D,{ }^{2} S,{ }^{2} P\right), 3 s^{2} 3 d^{2} D \\
3 s 3 p p^{2} P^{\circ} \\
2 s 2 p^{2}\left({ }^{2} D,{ }^{2} S,{ }^{2} P\right) \\
3 s 3 p^{3}\left({ }^{3} D^{\circ},{ }^{3} P^{\circ},{ }^{3} S\right), 3 p 3 d\left({ }^{3} P^{\circ},{ }^{3} D^{\circ}\right) \\
3 s 3 p^{2}\left({ }^{2} D,{ }^{2} S,{ }^{2} P\right), 3 s^{2} 3 d^{2} D\end{array}$ & $\begin{array}{l}3 s^{2} 4 s^{2} S \\
3 s 4 p^{1} P \\
2 s^{2} 3 s^{2} S, 2 s^{2} 3 d^{2} D, 2 s 2 p^{3} P^{\circ} 3 p\left({ }^{2} P,{ }^{2} D,{ }^{2} S\right), 2 s 2 p^{1} P^{\circ} 3 p\left({ }^{2} P,{ }^{2} D,{ }^{2} S\right) \\
3 p 4 s^{3} P^{\circ} \\
3 s^{2} 4 s^{2} S\end{array}$ \\
\hline
\end{tabular}

increases, as shown in Figure 3, and are important contributors to the recombination rates at higher temperatures (as shown later).

Although relatively few, the excited equivalent electron bound states of the $(e+$ ion) system may make important contribution to the total recombination rate coefficient due to extensive autoionizing resonances in their photoionization cross sections. Figure 4 presents the partial photoionization cross sections $\sigma_{\text {pr }}(g)$ into the ground state of $3 s^{2} 3 p\left({ }^{2} P^{\circ}\right)$ of $S$ IV, for two $S$ In bound states: the metastable $3 p^{4}\left({ }^{3} P\right)$ and another excited state $3 s 3 p^{3}\left({ }^{3} S^{\circ}\right)$. Photoionization threshold of the $3 p$ optical electron corresponds to the excited target ion states of $S$ IV, $3 p^{3}\left({ }^{4} S^{0}\right)$ and the $3 s 3 p^{2}\left({ }^{4} P\right)$ respectively, as shown by the

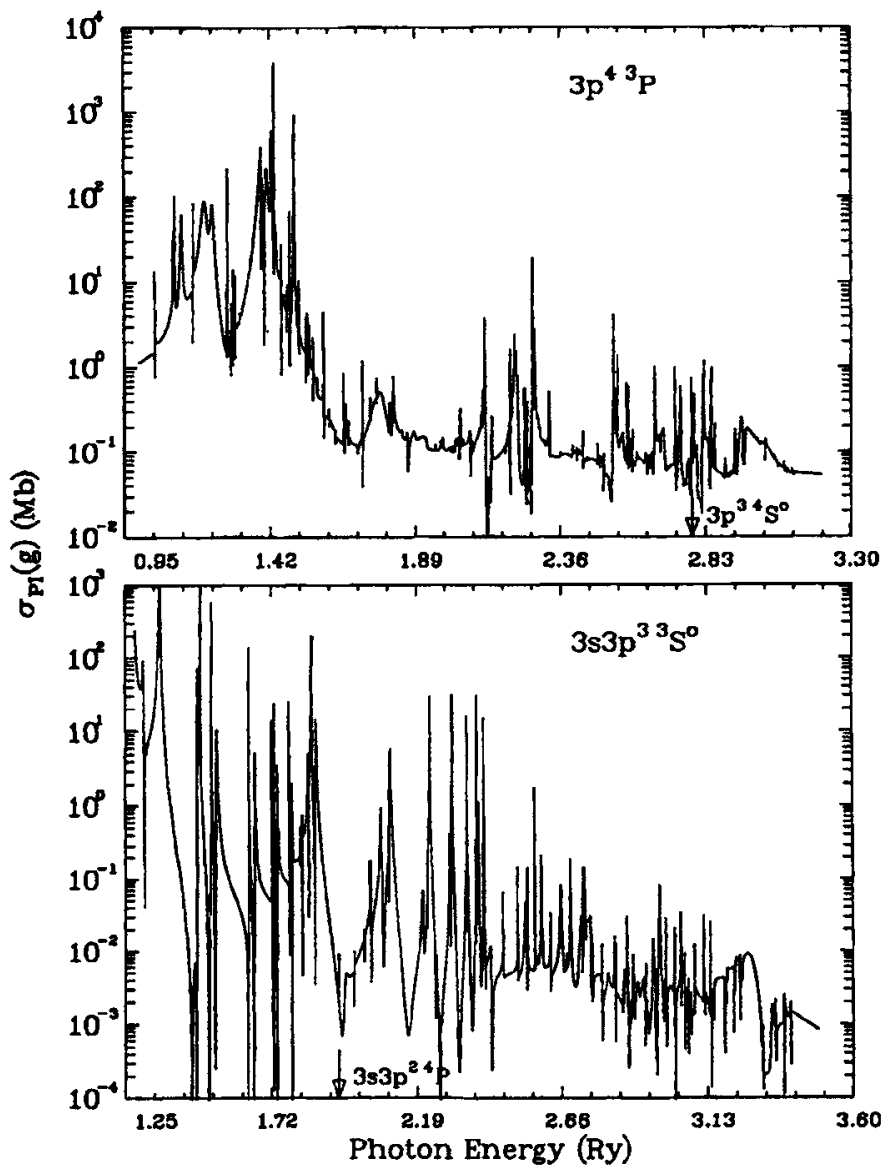

Fig. 4.-Partial photoionization cross sections, $\sigma_{\mathrm{p}}(\theta)$ of the metastable states $3 p^{4}{ }^{3} P$ (top panel), and $3 s 3 p^{3} S^{0}$ (bottom panet) of $\mathrm{S}$ in. The arrows indicate the energy of the lowest target threshold for ionization of the optical $3 p$ electron for these states. arrows in Figure 4. However, photoionization proceeds below these thresholds via autoionizing resonances coupled to the ground-state continuum and converging on to the excited states of $S$ IV. In the case of $3 p^{4}\left({ }^{3} P\right)$, the $3 p$ ionization threshold $3 p^{3}\left({ }^{4} S^{9}\right)$ lies quite high compared to the ground state $3 s^{2} 3 p\left({ }^{2} P^{\circ}\right)$ of $\mathrm{S}$ rv. Nonetheless, the region near the ground state is dominated by large resonances. There is a considerable drop in the partial $\sigma_{\mathrm{pr}}\left(\theta,{ }^{3} P\right)$ at $\sim 1.6$ rydbergs, corresponding to the onset of the $3 s 3 p^{2}\left({ }^{4} P\right)$ continuum which is more strongly coupled to the $3 p^{4}\left({ }^{3} P\right)$, i.e., photoionization flux is diverted into the ${ }^{4} P$ instead of contributing to photoionization into the ground state ${ }^{2} P^{o}$ of S IV. Similarly, the $3 s 3 p^{3}\left({ }^{3} S^{0}\right)$ cross section is generally enhanced in the region ${ }^{2} P^{0}-4 P$, although the ionization threshold of the $3 p$ electron is at $3 s 3 p^{2}\left({ }^{4} P\right)$. The partial photoionization cross sections are discussed in more detail in Nahar \& Pradhan (1993). The consequence of including a number of excited $(e+$ ion) states of this type is that the total recombination rate coefficient is significantly enhanced, particularly in the low-temperature region $T<10,000 \mathrm{~K}$.

We should like to emphasize the importance of complete resolution of resonances in the photoionization cross sections. The present photoionization cross sections are not from the OP, but are recalculated in a similar manner. The differences between the present cross sections and those of the OP are that the present calculations include (1) a larger number of bound states $i_{b}$ with larger value of $l$; (2) more extensive $C C$ wavefunction expansions for the target ions, which result in (a) additional bound states and $(b)$ more Rydberg and PEC resonances at higher energies; (3) resolution of resonances near the first ionization threshold with finer energy mesh; and (4) calculation of partial photoionization cross sections into each of the target states of the recombining ion included in the close coupling expansion. Better resolution of resonances near the ionization threshold is very important since it affects the recombination rate coefficients considerably, especially at low temperatures where a sharp rise, a low-temperature "bump," may occur (Nussbaumer \& Storey 1983). Large CC expansions, with more excited states of the target ion, provide more accurate rate coefficients at higher temperatures.

\subsection{Collision Strengths for Dielectronic Recombination}

For the high-n states of group B, where recombination proceeds mainly through DR, we employ the theory of DR developed by Bell \& Seaton (1985, hereafter BS). Below each target threshold, $S_{t} L_{t} \pi_{t}$, the Rydberg series of states $S_{t} L_{t} \pi_{t} v l$, where $v$ is the effective quantum number, are quasi-bound (i.e., lie above the first ionization threshold), and manifest themselves through autoionizing resonances. In the present work the DR calculations are carried out for the Rydberg series of resonances belonging to each target threshold $S_{t} L_{2} \pi_{t}$ that is dipole connected with the target ground state. Table 2 presents the 
dipole core transitions for the ions considered. We note expecially that the same CC wavefunction expansion is employed for both the photoionization and the DR calculations, thus ensuring consistency and accuracy.

In the BS theory, the DR probability, $P_{\alpha}^{S L \pi}$, for each channel $\alpha$ is obtained from the general scattering matrix, which includes radiative decay, and satisfies the unitarity condition. Both types of DR collision strengths, detailed ones with autoionizing resonances and resonance averaged, are obtained as described in NP3. The total DR collision strength, $\Omega(D R)$, for channel $\alpha$ is given by

$$
\Omega(\mathrm{DR})=\sum_{s, L, \pi} \sum_{\alpha} \frac{1}{2}(2 S+1)(2 L+1) P_{\alpha}^{S L \pi}(\mathrm{DR}) .
$$

All radiative and collisional channeis are coupled, so that autoionization or radiative decays in to excited target states are included in an $a b$ initio manner (autoionization into excited states was shown to be of importance by Jacobs et al. 1979). Along with the autoionization rates, the DR probability depends on the radiative transition probabilities ( $A$-values) for the dipole-allowed transitions in the recombining ion. These transition probabilities for the present work are obtained from oscillator strengths computed by the OP. The relevant $R$ matrix codes have been extended for the calculation of DR collision strengths, which are obtained as a continuous function of the effective quantum number $v$ relative to each target threshold. The DR collision strength shows characteristic peaks at the target thresholds and is related to the electronimpact-excitation (EIE) collision strengths, $\Omega(E I E)$, such that as the trapped fiux due to DR below an excited threshold is released at the threshold, the value of $\Omega(D R)$ should be equal to $\Omega$ (EIE). In the present work, the value of $\Omega(E I E)$ at the target threshold is calculated to provide a check against the peak value of $\Omega(\mathrm{DR})$ at that threshold (exceptions may occur in the presence of a resonance close to the excited threshold as discussed in NP3).

Figures 5-9 present $\Omega(D R)$ for $\mathrm{Si}$ I, Si II, Si Ix, S II, and S mI. For each ion the DR calculations start at effective quantum number $v=10$, as pointed by arrows in the figures, for the Rydberg series of states belonging to each excited target threshold $S_{t} L_{t} \pi_{t}$ that is connected via dipole transition to the ground state. The detailed and the resonance-averaged DR collision strengths are calculated. Figures $5,6,7,8$, and $9 a$ present the resonance-averaged collision strengths, $\langle\Omega(D R)\rangle$, for Si I, Si II, Si IX, S II, and S III, respectively. As expected, it can be seen that $\langle\Omega(D R)\rangle$ is very small in general up to about $v=10.0$, but rises quickly and peaks at the target threshold; the recombination probability increases as $\nu^{3}$. Among the ions considered, $\mathrm{Si}$ Ix, which has the maximum ion charge, shows (Fig. 7) low values of $\Omega(\mathrm{DR})$ for the core transitions from higher states in $n=3$ complex indicating negligible high- $n$ DR contributions due to these transitions.

In Figures 5-9a, the filled circles correspond to the collision strength for electron impact excitation, $\Omega$ (EIE), at the target thresholds, obtained using the same $\mathrm{CC}$ expansion as for $\Omega(D R)$. This provides the check that at threshold both the $\Omega(D R)$ and the $\Omega(E I E)$ are equal. In some instances the two values may be different, as mentioned before, if a resonance
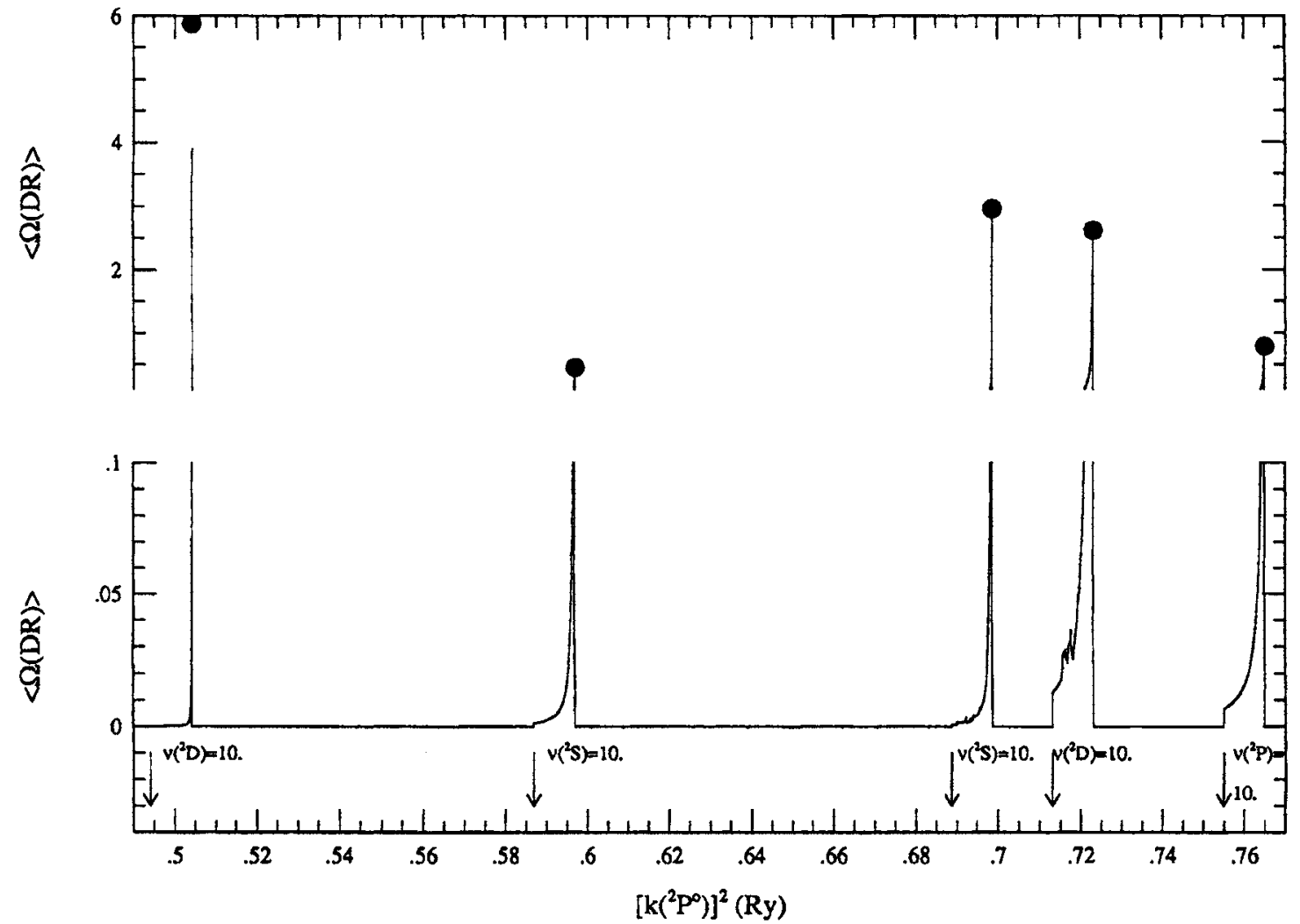

Fis. 5. - Resonance-averaged DR collision strength, $\langle\Omega(D R)\rangle$, for $e+S_{i}$ if $\rightarrow S_{i}$. Calculation of $\langle\Omega(D R)\rangle$ starts at the positions indicated by the arrows where the effective quantum number $v=10.0$ for the Rydberg series $S_{t} L_{t} \pi_{t} v l$ belonging to each of the target threshold $S_{t} L_{t} \pi_{t}={ }^{2} D,{ }^{2} S,{ }^{2} S,{ }^{2} D$, and ${ }^{2} P$ of $S i n$ in (see Table 1

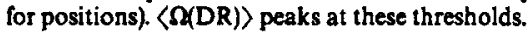



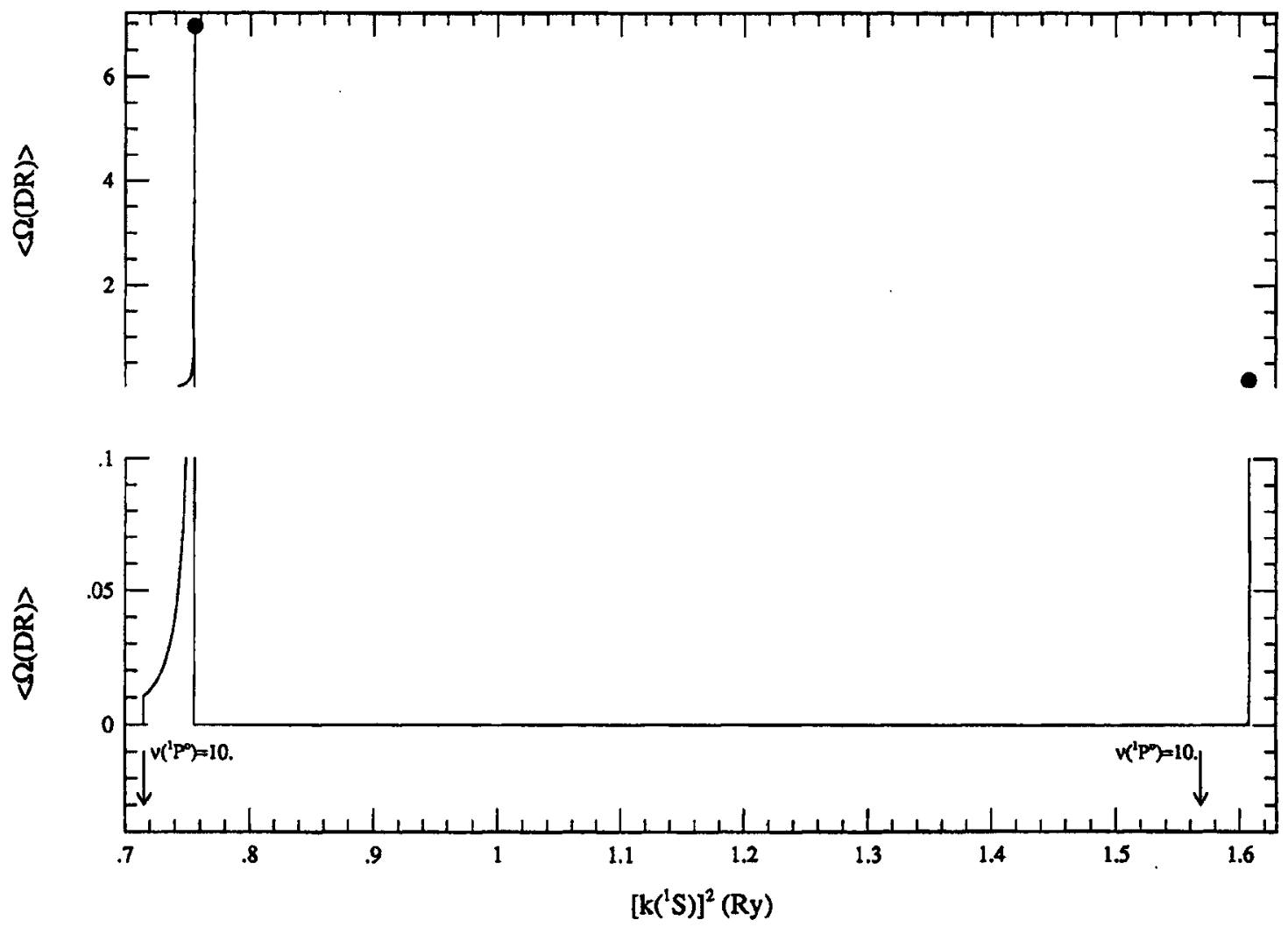

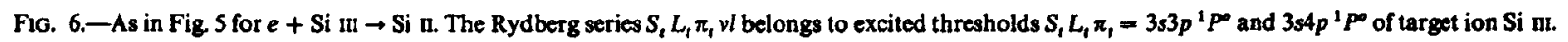
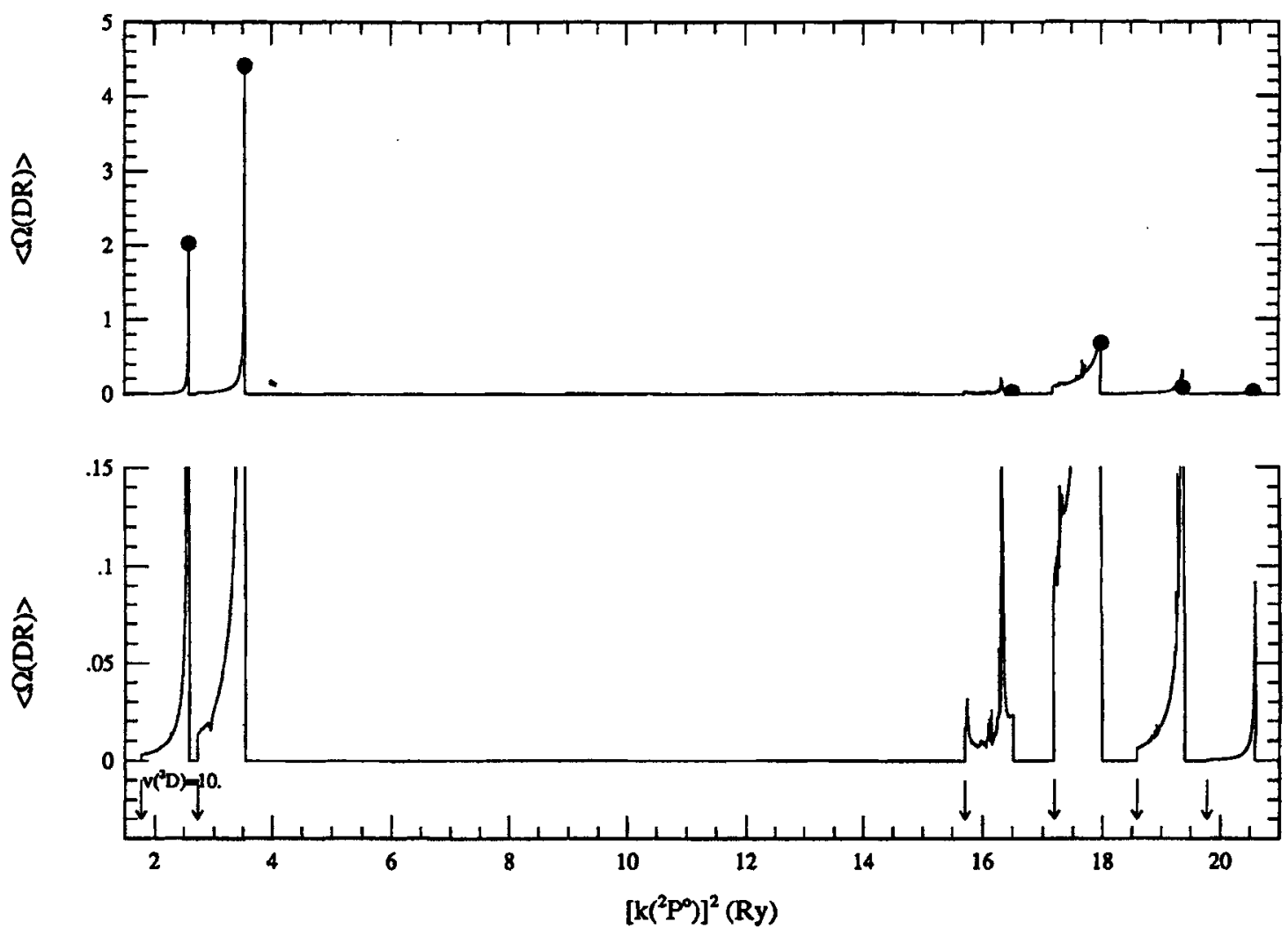

Fia. 7.-As in Fig. S, for $e+S i x \rightarrow$ Si $x$. The Rydberg series $S_{t} L_{t} \pi_{t} v l$ belongs to excited thresholds $S_{t} L_{t} \pi_{t}={ }^{2} D,{ }^{2} S,{ }^{2} P$ of the $n=2$ complex and ${ }^{2} S,{ }^{2} D,\left({ }^{2} P,{ }^{2} D\right.$, $\left.{ }^{2} S\right),\left({ }^{2} S,{ }^{2} D,{ }^{2} P\right)$ of the $n=3$ compiex of target ion Si $x$. States within parentheses are treated as degenerate in energy. 

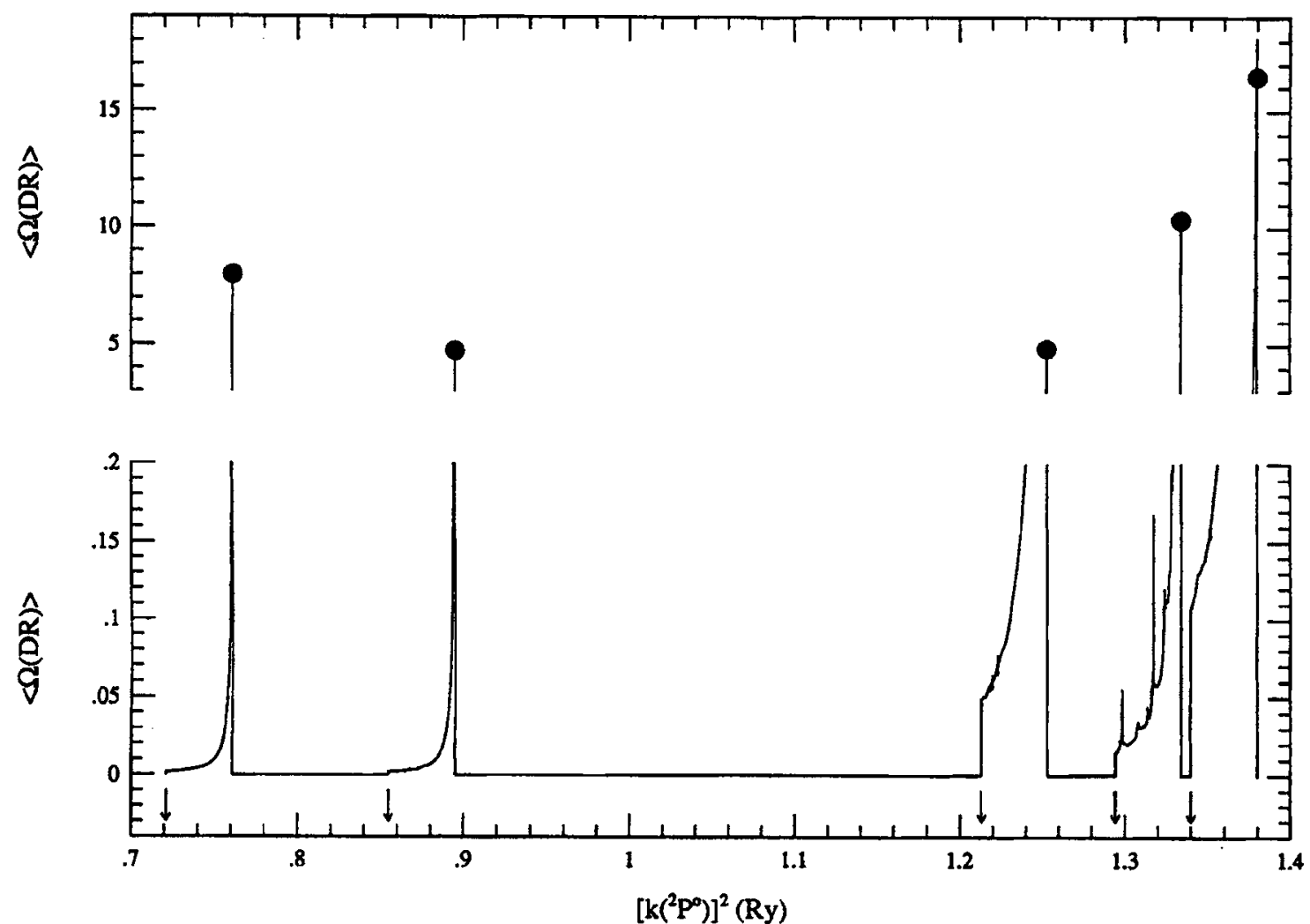

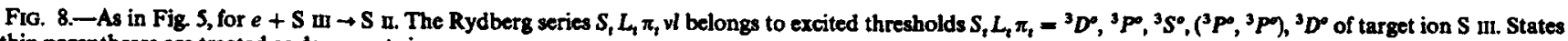
within parentheses are treated as degenerate in energy.

exists close to the threshold, for example, the $\Omega(D R)$ peak at the ${ }^{2} D$ threshold of Si $n$ in Figure 5 is lower than $\Omega(E I E ;$ $\left.{ }^{2} P^{\circ} \rightarrow{ }^{2} D\right)$

Although full computational details are given in NP3, a few general points might be mentioned. In case of target states that lie close together in energy, they are treated as degenerate, e.g., for Si IX the target states $2 s 2 p^{22} S, 2 s 2 p^{22} P$ are set degenerate. Below some target thresholds the resonance-averaged $\langle\boldsymbol{\Omega ( D R})\rangle$ are superposed with small resonance structures, as in Figure 7 for Si IX. The resonances below a target threshold can consist of those belonging to that immediate threshold, as well as to those belonging to the higher target thresholds, usually the next higher one. In the present procedure of resonance averaging, the resonances belonging to the first threshold are averaged over, but not from the higher ones, and hence those may show up in $\langle\Omega(D R)\rangle$. These can be easily smoothed over by eliminating the channels of higher thresholds. However, that violates the unitarity of the $S$-matrix to some extent and may affect the peak value of $\langle\Omega(D R)\rangle$. In general there are only a few such resonances in the energy region where $\langle\Omega(D R)\rangle$ is calculated, and only a small uncertainty is thereby introduced in the recombination rate coefficients. In the present work, the resonance-averaged $\langle\Omega(D R)\rangle$ are used to calculate the recombination rate coefficients, as these are easier to convolute with the Maxwellian than the detailed $\Omega(D R)$ with numerous narrow resonances.

Figure $9 b$ shows the detailed $\Omega(D R)$ with autoionizing resonances for the recombination of $e+\mathrm{S}$ iv $\rightarrow \mathrm{S}$ III. The separation of resonances gets narrower with effective quantum number of the Rydberg series, and the resonances converge on to the corresponding target threshold. As the resonances approach the threshold, the background also rises. An expanded picture of the DR resonances (that appear as dashed lines in Fig. $9 b$ ) is presented in Figure $9 c$ for the Rydberg resonances belonging to the first target threshold ${ }^{2} D$ of $\mathrm{S}$ In. Detailed resonances for the $n$-complexes, as specified in the figure from $n=20$ onward, can be seen as repeated patterns which get narrower and higher with each successive $n$. The present method enables the calculations of detailed DR collision strengths (Fig. 9c) for the first time.

\section{RESULTS AND DISCUSSION}

Figures 10-14 present the total recombination rate coefficients, $\alpha_{R}(T)$, over a wide range of temperatures, $1.0 \leq \log _{10}$ $(T) \leq 9.0$ for recombination of Si $\mathrm{l}, \mathrm{Si} \mathrm{I}, \mathrm{S}$ II, S mi, and Si Ix, respectively. One distinctive feature of the total recombination rate coefficients, $\alpha_{R}(T)$, is the presence of a minimum in the intermediate-temperature region beyond which $D R$ is usually the dominating process. While all processes are considered in a unified manner, the general pattern of the $\alpha_{R}(T)$ corresponds, at low temperatures, to the contribution from recombination to low- $n$ bound states of the $e+$ ion system, i.e., primarily $\mathbf{R R}$, superposed with the low-temperature DR-type contributions from near threshold resonances. Before the onset of the high temperature DR, $\alpha_{R}(T)$ decreases with $T$, leading to a minimum at an intermediate temperature. As mentioned earlier, three separate sets of calculations in different approx- 

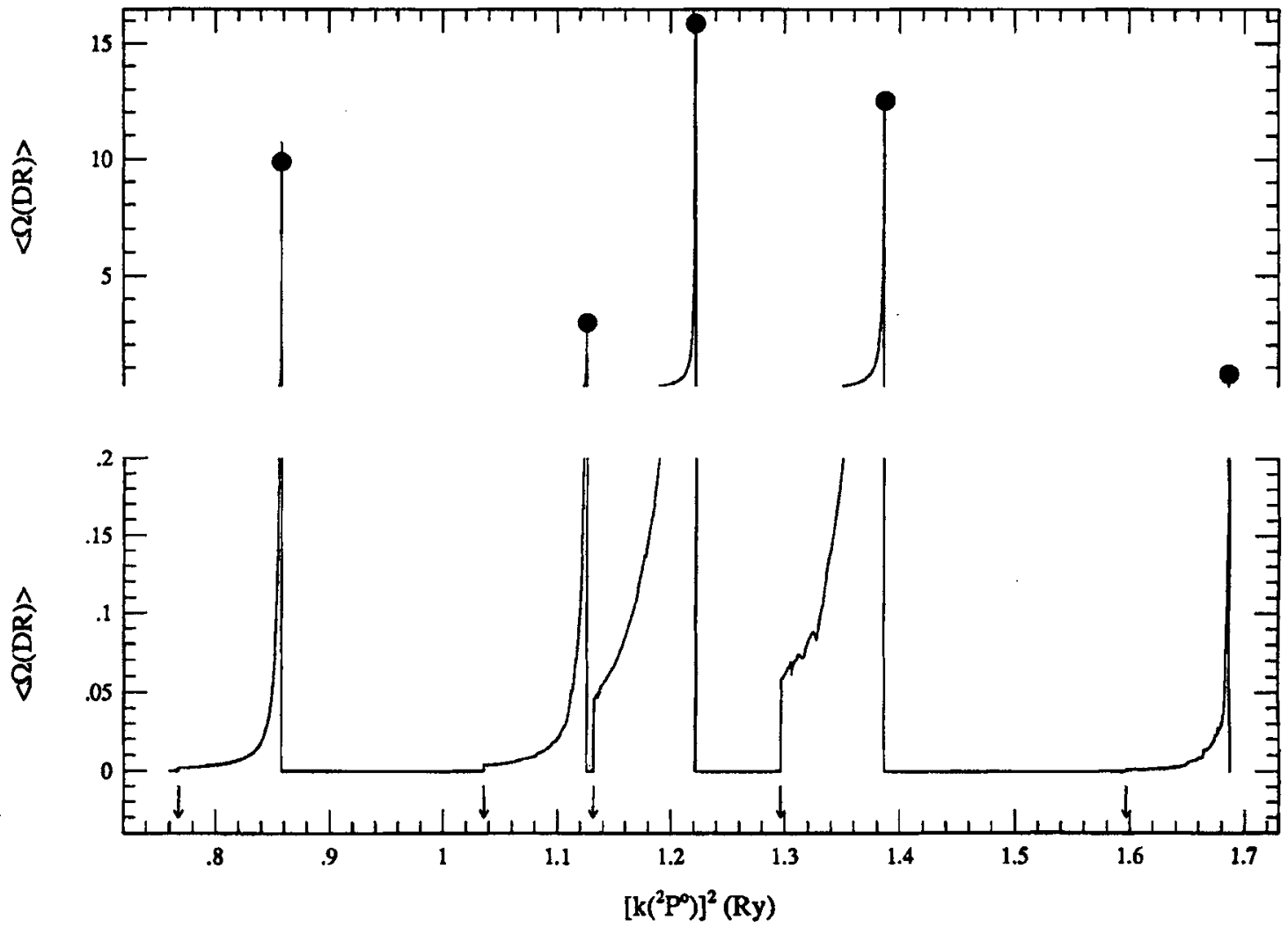

Fio. $9 a$
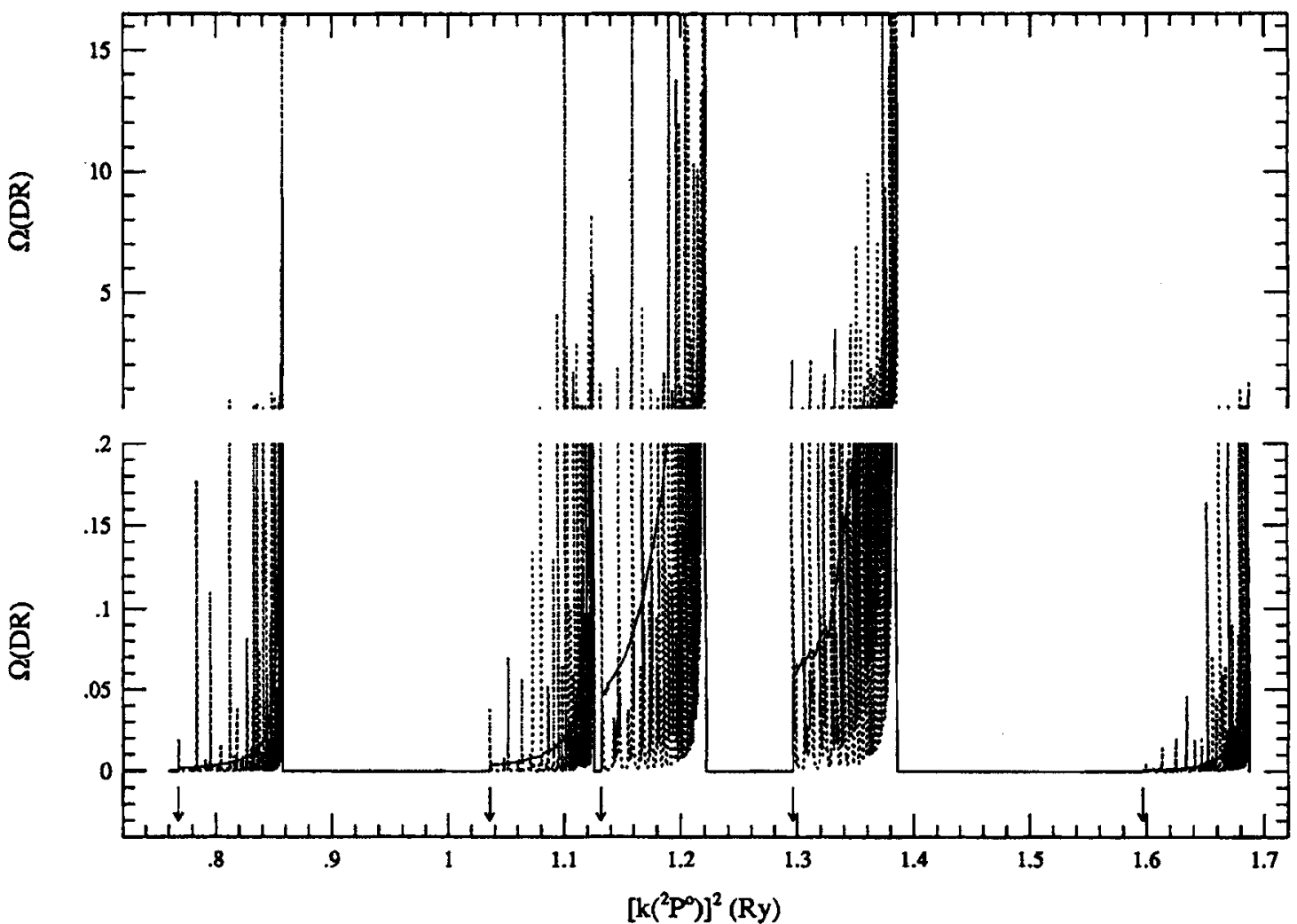

Fig. $9 b$

Fic. 9.- (a) As in Fig. S, for $e+S$ IV $\rightarrow S$ III. The Rydberg series $S_{t} L_{t} \pi_{t} v$ l belongs to excited thresholds $S_{t} L_{t} \pi_{t}={ }^{2} D,{ }^{2} S,{ }^{2} P,{ }^{2} D,{ }^{2} S$ of target ion $S$ rv. (b) Detailed DR collision strength with autoionizing resonances. The DR resonances converge on to the threshold it belongs to while the background rises. (c) Fully resolved DR collision strength, on an expanded energy scale with autoionizing resonances that appear as lines in (b). These correspond to the Rydberg series converning on to the first excited target threshold ${ }^{2} D$. Fine details within each $n$ complex of resonances from $n=20$ onward are shown. The patterns are repeated and become narrower as the effective quantum number increases. The DR collision strength corresponds to the emitted photon spectrum. 


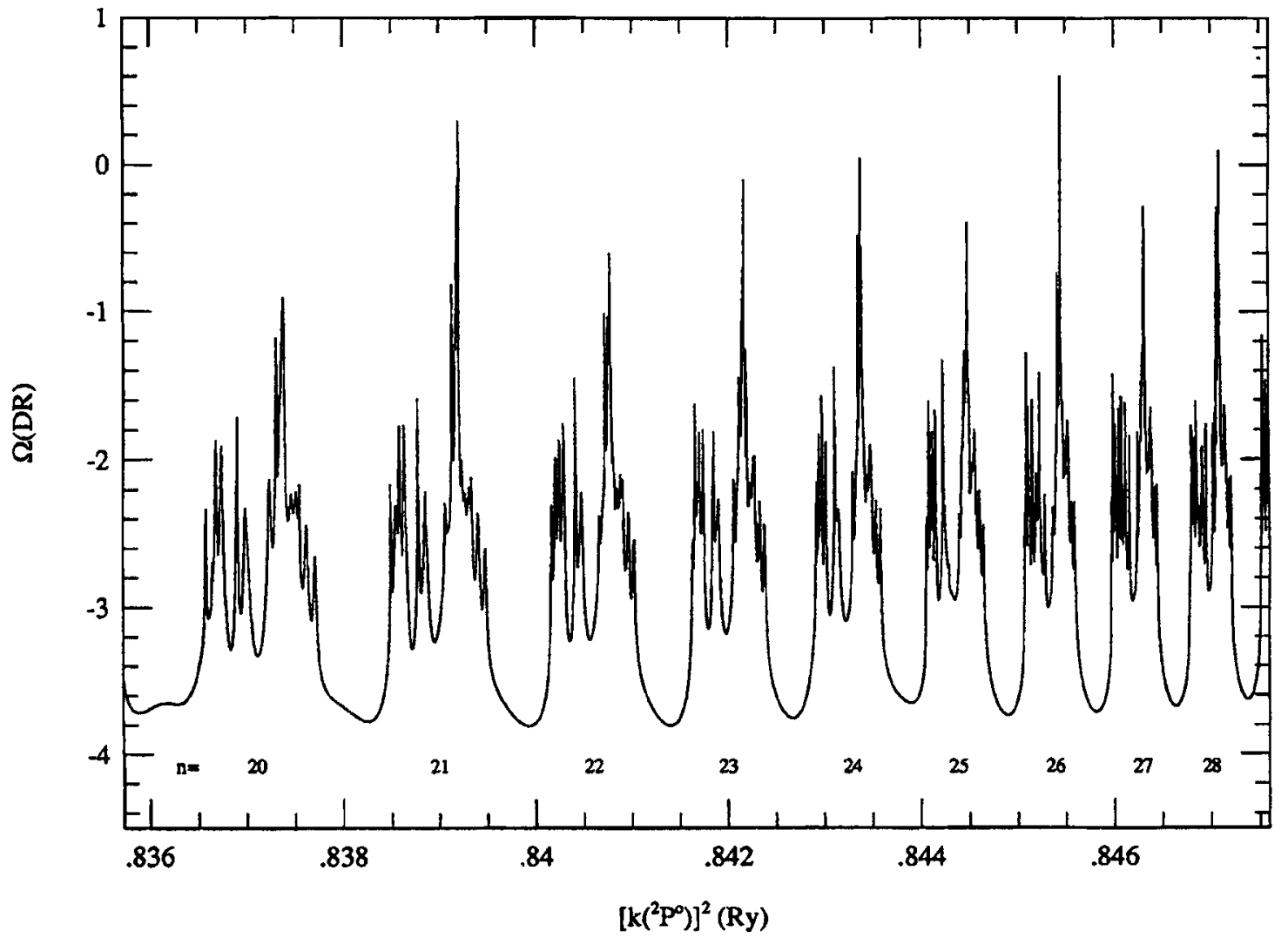

Fig. $9 c$

imations have heretofore been carried out to obtain the total recombination rate; direct $R R$, low- $T$ DR and high- $T D R$. The sum of the three different rates can be most uncertain in the region of overlap where no single process dominates the total recombination rate. This is likely to be in the intermediate- $T$ region where the minimum in $\alpha_{R}(T)$ occurs. The present work obtains the behavior of $\alpha_{R}(T)$ in this intermediate- $T$ region consistent with the low- $T$ and the high- $T$ ranges.

Figure 10 presents total recombination rate coefficients (solid curve) for $e+\mathrm{Si} \mathrm{I} \rightarrow \mathrm{Si}$ i. Comparison is made with three other calculations: $\mathbf{R} R$ rate coefficients by Aldrovandi \& Pequignot (1973, hereafter AP) (dashed curve), low-temperature DR rate coefficients by Nussbaumer \& Storey (1986, hereafter NS) (dotted curve), and high-temperature DR rate coefficients by Jacobs et al. (1977) (dash-chained curve). The present $\alpha_{R}(T)$ differs from the high-temperature DR rates of Jacobs et al. in the region $10,000-100,000 \mathrm{~K}$ where the present values are lower by a factor of 2.5 around $50,000 \mathrm{~K}$.

Figure 11 presents the total $\alpha_{R}(T)$ for the recombination of $e+\mathrm{Si} \mathrm{III} \rightarrow$ Si II (solid curve) and compares with other works, by AP (dashed curve), low-temperature DR rates by NS, and high-temperature DR rates by Jacobs et al. (1977) (dashchained curve). At low temperatures the rates by AP overestimate the recombination rate, while for the rest of the temperature range the sum of the three rates appears to be in fair agreement with the present $\alpha_{R}(T)$ for $\mathrm{Si}$ II except near the high-T peak where DR rates of Jacobs et al. are somewhat higher. The low-T DR rate coefficients by NS show variation that are not seen in the present results. Although the present values are obtained in a consistent manner for the entire range of temperatures, the values at very low temperatures may have high uncertainties in cases where one or more resonances might be present close to the ionization threshold. The precise position and full resolution of resonances near threshold are therefore very important, since even a small shift in the energy of a single resonance can introduce large uncertainty in the rates, up to perhaps $50 \%$ for $T<1000 \mathrm{~K}$.

Figure 12 presents the total $\alpha_{R}(T)$ for the recombination of $e+\mathrm{S}$ III $\rightarrow \mathrm{S}$ II (solid line). Comparison is made with the rate coefficients for RR by AP (dashed curve), high-temperature DR rates (dash-chained curve) by Jacobs et al. (1979), and also the high-temperature DR rates (asterisks) by Badncll (1991). As for S II, the AP data for RR overestimate the recombination rate at low temperatures. Present rates are in good agreement with the high-temperature DR rate coefficients by Jacobs et al. and up to a factor of 2 lower than those of Badnell at the high- $T$ peak. In their paper discussing the ionization structure and abundance depletions, Spitzer \& Fitzpatrick (1993) estimate the total recombination rate coefficient for $S$ II, at $6000 \mathrm{~K}$, to be $4 \times 10^{-12} \mathrm{~cm}^{3} \mathrm{~s}^{-1}$. This is nearly one and one-half times the $R R$ rate coefficient given by AP, $2.7 \times 10^{-12} \mathrm{~cm}^{3} \mathrm{~s}^{-1}$, but not far from the present calculated value which is $4.2 \times 10^{-12}$ $\mathrm{cm}^{3} \mathrm{~s}^{-1}$. Thus Spitzer \& Fitzpatrick appear to have correctly deduced that there might be a considerable low- $T$ enhance- 


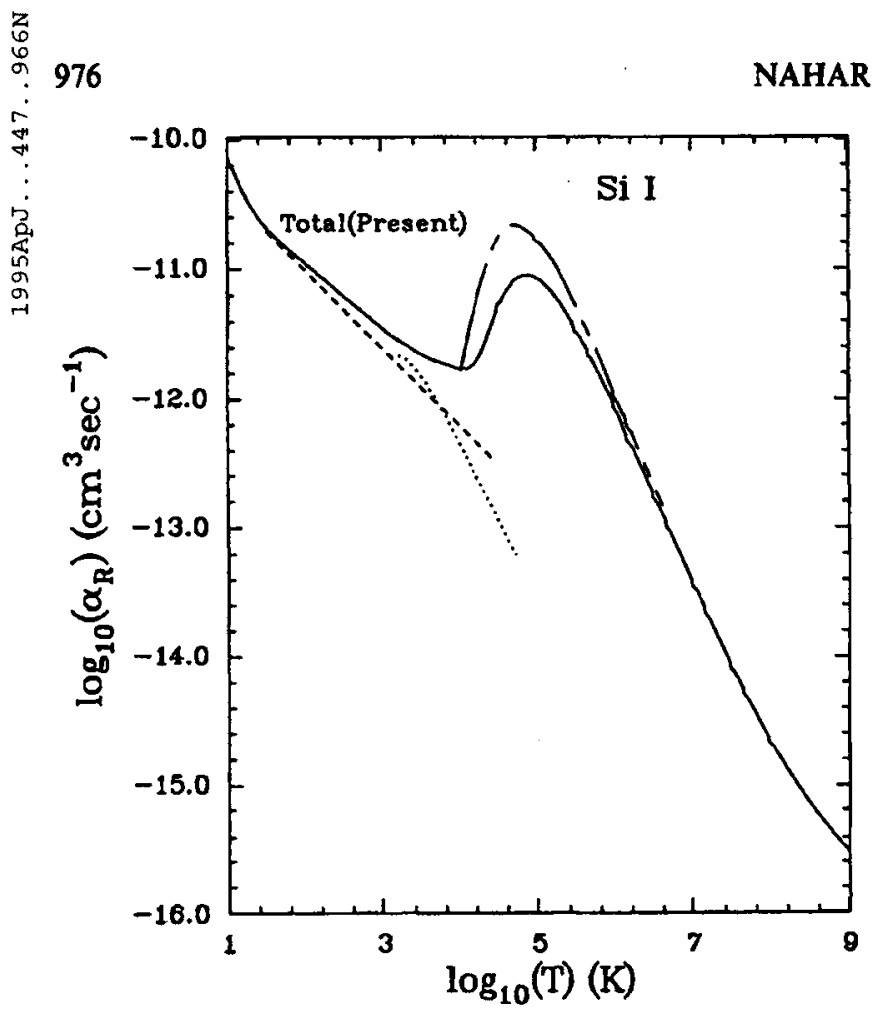

Fuc. 10.-Total recombination rate coefficients, $\alpha_{p}$, for $e+\operatorname{Si~} 11 \rightarrow \mathrm{Si}$ I (solid curve). The dashed curve is due to RR only by Aldrovandi \& Pequignot (1973), the dotted curve due to low-temperature DR by Nussbaumer and Storey (1986), and the dashed-chain curve due to high temperature DR by Jacobs et al. (1977).

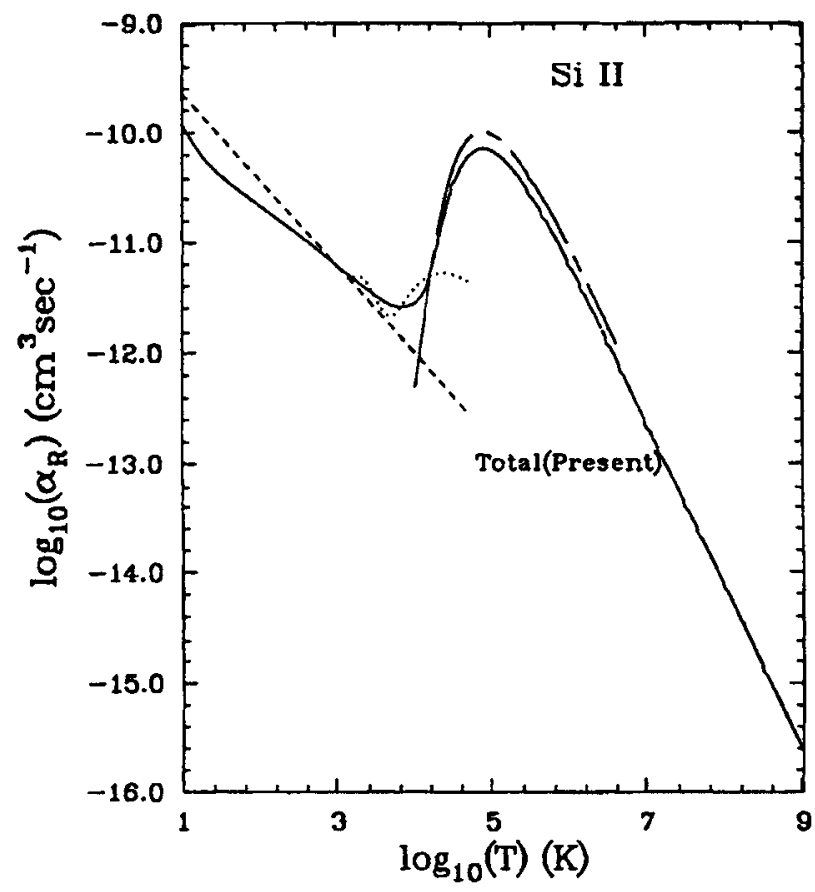

Fig. 11..-As in Fig. 10, for the recombination of $e+\mathrm{Si} \mathrm{ul} \rightarrow \mathrm{Si} \mathrm{d}$

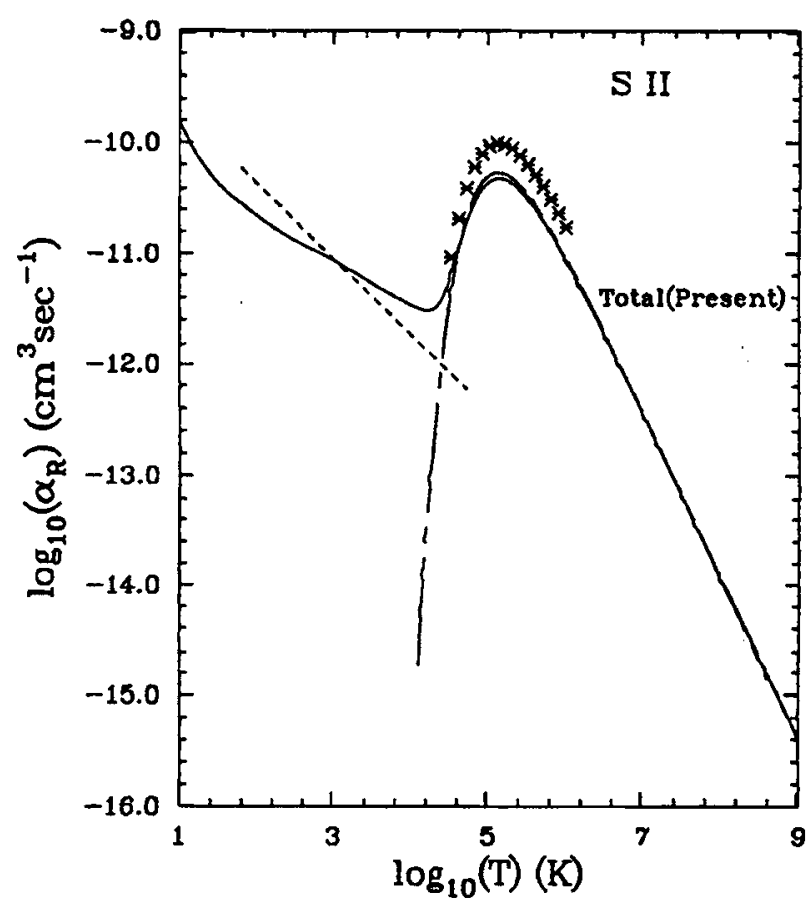

Fig. 12-Total recombination rate coefficients, $\alpha_{R}$, for the recombination of $e+S$ III $\rightarrow$ S II. The dashod curve is due to $R R$ only by Aldrovandi \& Pequignot (1973), the dashed-chain curve due to high temperature DR by Jacobs et al. (1979), and the asterisks are high-temperature DR rate coefficients by Badnell (1991).

ment in the rate due to low-lying resonances with significant implications for the sulfur abundance in the Galactic ISM. The recombination rates for $S$ I presented herein have been improved over those presented in NP3. In the earlier calculations of $\Omega(D R)$ we excluded the interference effects of the channels of the higher thresholds (that do not couple to the dipole allowed core transitions) to smooth out the $\langle\Omega(D R)\rangle$ curve, with some effect on the $(\Omega(D R)\rangle$ peaks at two target thresholds. In the present calculations the unitarity of the $S$ matrix is fully conserved, although it leads to small features in an otherwise smooth $\langle\Omega(D R)\rangle$. Also, in calculating the present rates the autoionizing resonances near the threshold are resolved with a fine energy mesh, which results in a significant increment in the rate at low temperatures.

Figure 13 presents the total $\alpha_{R}(T)$ for the recombination of $e+S$ iv $\rightarrow S$ in (solid line). Comparison with other works shows that for $\mathbf{S ~ m l}$, the RR rates by AP (dashed curve) underestimate the recombination rates considerably. While the hightemperature peak occurs at the same temperature, the present recombination rates are lower than those by Jacobs et al. (1979) (dashed-chained curve) and Badnell (1991) (asterisks) by about $50 \%$ at the peak.

Figure 14 presents the total $\alpha_{R}(T)$ for the recombination of $e+$ Si X $\rightarrow$ Si IX (solid line). Unlike other ions considered here, Si IX shows some interesting features in the recombination rates, characteristic of $e$-ion recombination to the carbonsequence ions reported in NP3. There is a low-temperature "bump" caused by a few low-lying and wide autoionizing resonances in the photoionization cross sections of the equivalent electron states of Si $\mathrm{IX}$, such as $2 s^{2} 2 p^{2}\left({ }^{3} P,{ }^{1} D\right), 2 p^{4}\left({ }^{3} P,{ }^{1} D\right)$, $2 s 2 p^{3}\left({ }^{3} P^{0},{ }^{3} D^{0}\right)$. These low-lying autoionizing resonances may 
enhance the recombination rates at low temperatures considerably above the "background" recombination rate (as reflected in the RR rate only). Existence of such a low-temperature bump for carbon-like $\mathrm{O}$ III was reported by Nussbaumer \& Storey (1983) and was confirmed by our unified calculations (NP2, 1992). In addition to the low- $T$ bump, there are two small but distinct bumps in $\alpha_{R}(T)$ at high temperatures due to DR from high- $n$ and low- $n$ resonances. The RR rate coefficients by AP (dashed curve) are considerably lower, whereas the high-temperature rate coefficients by Jacobs et al. (1977) (dashchained curve) are in reasonable agreement with the present values. Our study of the recombination rates for carbon isoelectronic sequence (NP3), indicates that the high- $T$ bump tends to diminish with increasing ion charge. Present rates for Si IX calculated with a 23-state expansion are consistent with the other highly charged ions, such as Al viII and S IX (all rate coefficients will be presented in a supplementary publication). We may point out that Shull \& Steenberg (1986) fits for the DR rates of $\mathrm{Si} \mathrm{IX}$, as shown in Figure 8 in NP3, from the original values of Jacobs et al. (1977) are incorrect by an order of magnitude.

One of the most important considerations in the close coupling approximation is the convergence of the results as a function of the eigenfunction expansion in terms of the number of target ion states. In the present work we study the convergence for the recombination of $e+\mathrm{Si} \mathrm{x} \rightarrow \mathrm{Si}$ Ix. The earlier recombination rates (NP3), for Si IX corresponded to an eigenfunction expansion of eight terms of Si $\mathrm{X}$ from the $n=2$ complex (Nahar \& Pradhan 1992), while in the present work we have employed a 23-state expansion including the $n=3$ complex (Table 1). The larger Si $x$ expansion resulted in more bound states of Si IX below the ionization threshold, compared to the eight-state expansion, and eight additional dipole allowed core transitions with $\Delta n \neq 0$, for a total of 11 core transitions including the $\Delta n=0$ transitions (Table 2). Both of these factors contribute to some enhancement in the recombination rates and introduction of a second small bump in the high- $T$ region. Coupling of additional channels in the present work has reduced the dip found before the low- $T$ bump. While there are some differences between the eight $\mathrm{CC}$ and the $23 \mathrm{CC}$ results for the total rate coefficients, these are small enough to indicate that both the low- $n$ and the high- $n$ contributions have converged. Of the two small high- $T$ bumps the first one is due to DR contribution from the high- $n$ resonances $(n>10)$, which remains about the same in magnitude for both the eight $\mathrm{CC}$ and the $23 \mathrm{CC}$ calculations. This can also be inferred from Figure 7 showing fairly low values for high-n $\Omega(D R)$ due to core transitions from states in the $n=3$ complex. Figure 15 shows the photoionization cross sections for the $2 s^{2} 2 p^{2}{ }^{3} P$ ground state (bottom panel) and the excited $2 p^{3}\left({ }^{2} D^{\circ}\right) 3 p^{1} D$ state (top panel), respectively. The arrow position indicates the highest core state of the $n=2$ complex $2 p^{32} P^{\circ}$. Expanding the eigenfunction basis set from eight to 23 target states does not particularly enhance the background cross sections of the ${ }^{3} P$ ground state; there is little resonance structure between the $n=2$ and the $n=3$ complexes. However, the excited states' cross sections are considerably enhanced due to the PEC and the Rydberg type resonances, indicating strong coupling with the $n=3$ complex. The fact that there are a large number of excited bound states, all with PEC features in their photoionization cross sections, is responsible for the second bump at high $T$, and consequent enhancement in the total recombination rate coefficient. This is the first study of the role of PEC resonances in the $e$-ion recombination process.

\section{CONCLUSION}

We summarize the reported calculations of the unified recombination rate coefficients as follows:

1. A new, unified treatment of e-ion recombination is applied to obtain accurate, total rate coefficients of $\mathrm{S}$ and $\mathrm{Si}$ ions over all temperatures for low-density plasmas.

2. The method is an extension of the Opacity Project work on photoionization, extended to include the DR process as in electron-ion scattering, employing the theory developed by Bell \& Seaton (1985) (see also Pradhan \& Seaton 1985).

3. The treatment entails the calculation of a large number of detailed partial photoionization cross sections and DR collision strengths, both calculated with the same eigenfunction expansion for the recombining target ion, thus ensuring consistency and accuracy.

4. The general form of the total recombination rate coefficients shows the presence of distinct "bumps," at low $T$ and at high $T$, attributable to different sets of autoionizing resonances.

5. Although the overall accuracy of the rate coefficients should be the same as for the usual photoionization and electron-ion scattering calculations, approximately $10 \%-15 \%$, the rate coefficients at very low temperatures may be more uncertain, up to $50 \%$ for $T<1000 \mathrm{~K}$, due to near-threshold resonances.

6. Partial recombination rate coefficients have also been calculated for all $N_{\text {bnd }}$ (Table 1) bound states of each ion. Partial recombination rates may be useful in the analysis of recombination line spectra (e.g., Liu et al. 1994), arising from recombination to and radiative cascade from, specific excited states.

7. The present recombination rate coefficients are valid for low-density plasmas, where the excited population may be neglected. While the $(e+$ ion) continuum states not coupled to the ground state of the recombining ion have been excluded from the present work in LS coupling, these may make a small but significant contribution owing to autoionization in intermediate coupling (Nussbaumer \& Storey 1983). At very low temperatures it may be necessary to consider the nearthreshold resonances by including relativistic effects that manifest in (1) the resulting fine structure and (2) resonances forbidden to autoionize in pure LS coupling. Work is in progress on these two areas, as well as on recombination from excited states, particularly the metastable states (a discussion of some associated processes is given in Seaton \& Storey 1976, p. 133). For highly charged ions the effect of radiation damping in photoionization is being investigated (as discussed by Sakimoto, Terao, \& Berrington 1990).

8. The electronically accessible Opacity Project database, TOPBASE (Cunto et al. 1983), contains total photoionization cross sections which, for reasons discussed in $\$ 2.1$, may not yield accurate recombination rate coefficients. In addition, a complete treatment requires the inclusion of DR which dominates high-energy recombination.

9. The calculation of unified recombination rates is rather laborious and computationally demanding but, when completed for a large number of atoms and ions, should enable the substitution by a single $e$-ion recombination rate coefficient the different types of $R R$ and $D R$ rates currently employed in astrophysics and other plasma applications.

10. The total and partial rate coefficients are presented in tabular form in Nahar (1995) over the temperature range $10 \leq T \leq 10^{\circ} \mathrm{K}$, at $\Delta \log _{10} T=0.1$, for the ions $\mathrm{C}$ II, Si I, Si II, $\mathrm{S}$ II, S II, C I, N II, O III, F IV, Ne v, Na vi, $\mathrm{Mg}$ vII, Al viI, 


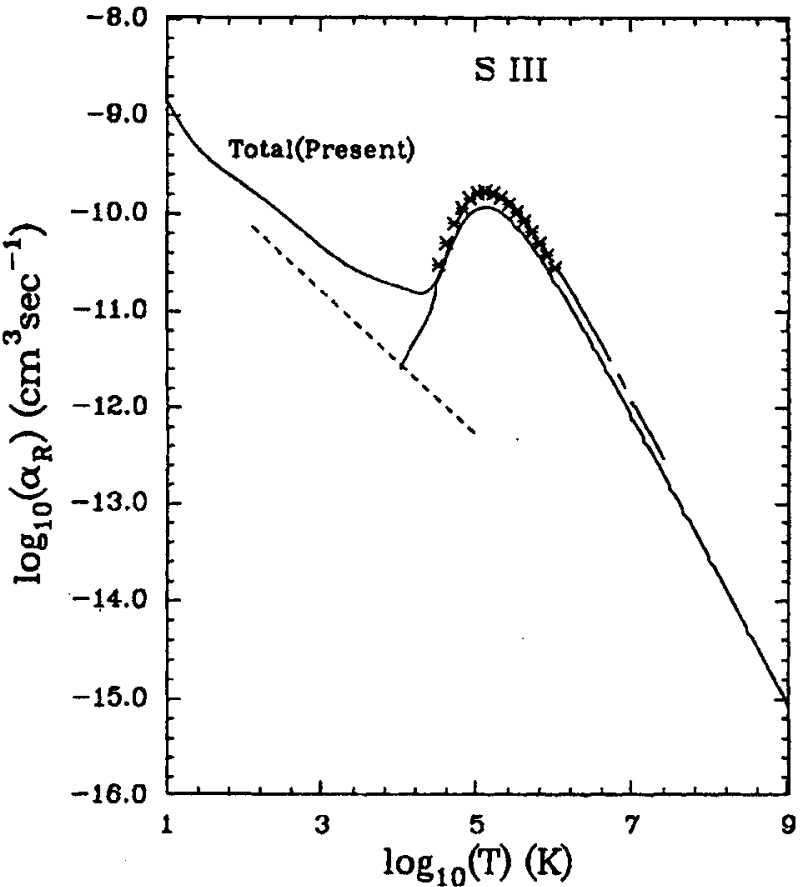

Fig. 13.-As in Fig. 12, for the recombination of $e+S \mathrm{sv} \rightarrow \mathrm{S} \mathrm{m}$

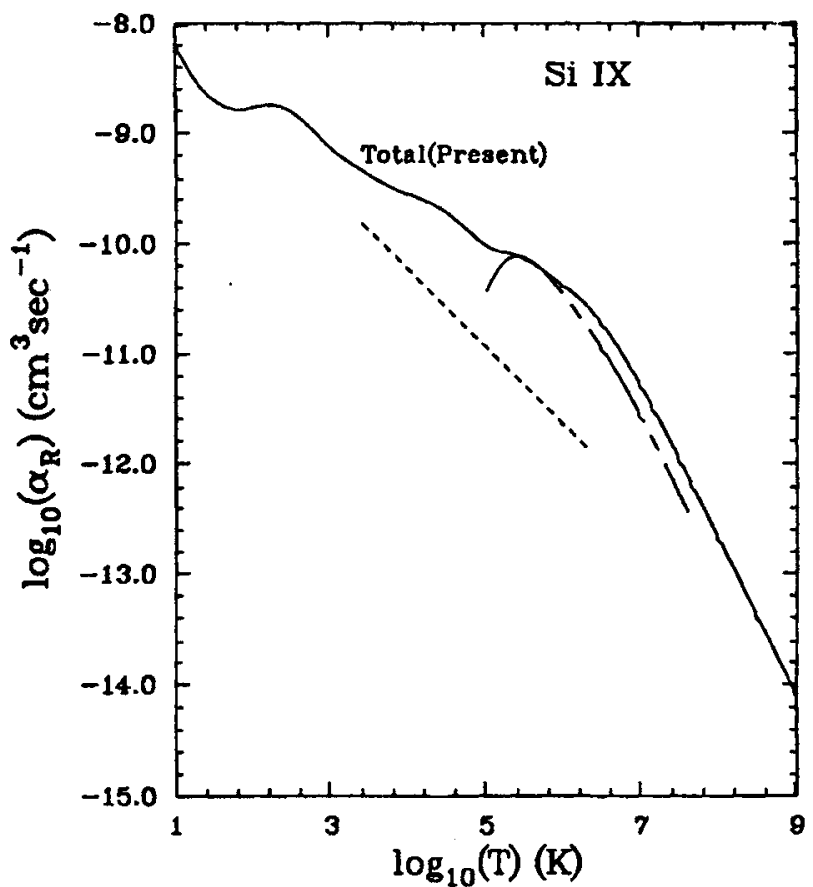

Fio. 14.-Total recombination rate coefficients, $\alpha_{k}$, for the recombination of $e+\mathrm{Si} x \rightarrow \operatorname{Si~Ix}$. The dashed curve is due to RR only by Aldrovandi \& Pequignot (1973), and the dashed-chain curve due to high temperature DR by Jacobs et al. (1977)

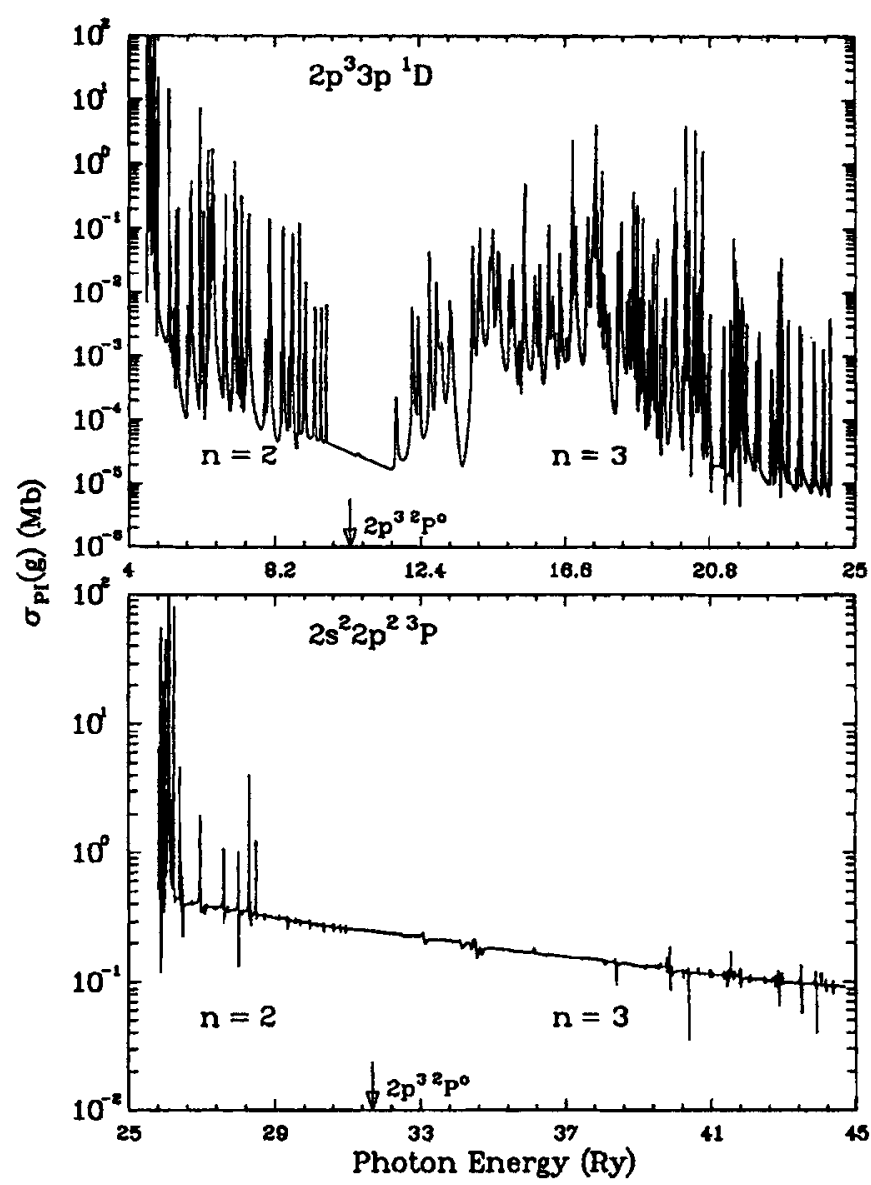

FIG. 15.-Partial photoionization cross sections, $\sigma_{m}(g)$, of the ground state $2 s^{2} 2 p^{2}{ }^{3} P$ (bottom panel) and the excited state $2 p^{3}\left({ }^{2} D^{1}\right) 3 p^{1} D$ (top panel) of Si $\mathrm{XX}$ into the ground state ${ }^{2} P^{\circ}$ of Si $X$. Resonances belonging to the target thresholds of the $n=2$ complex and the $n=3$ complex are shown. The arrows point to the energy of the highest target state $2 p^{32} p^{\circ}$ in the $n=2$ complex.

Si IX, and S XI. All calculations are carried out as described here.

We would like to thank Professor Lyman Spitzer for drawing our attention to the need for accurate recombination rates for $\mathbf{S}$ and $\mathrm{Si}$ ions of importance in the ISM studies. The authors would like to thank C. Mendoza for providing the CC target ion expansion for Si in. We also thank D. Verner and G. J. Ferland for discussions. This work was supported by NASA (NAGW-3315) under the Long Term Space Astrophysics (LTSA) program, and the NSF (PHY-9421898) grant for the Iron Project. The calculations were performed on the Cray Y-MP at the Ohio Supercomputer Center in Columbus.

\section{REFERENCES}

Aldrovandi, S. M. W., \& Pequignot, D. 1973, A\&A, 25, 137 (AP) Badnell, N. T. 1991, ApJ, 379, 356

Bell, R. H., \& Seaton, M. J. 1985, Phys. B, 18, 1631 (BS)

Berrington, K. A., Burke, P. G., Butler, K., Seaton, M. J., Storey, P. J., Taylor, K. T. \& Yan, Y. 1987, J. Phys. B, 20,6379
Cunto, W., Mendoza, C. Ochsenbein, F. \& Zeippen, C. J. 1993, A\&A, 275, L5 Jacobs, Y. L Davies, J Keppler, P. C \& Blaha M 1977 ApJ, 215, 690 Jacobs, V. L., Davies, J., Rogerson, J. E., \& Blaha, M. 1979, ApJ, 230, 627 Liu, X. W., Storey, P. J., Barlow, M. J., \& Clegg, R. E. S. 994, MNRAS, in press 
\begin{tabular}{l}
$z$ \\
6 \\
$\swarrow$ \\
\hdashline
\end{tabular}

Nahar, S. N. 1995, ApJS, in press

Nahar, S. N. \& Pradhan, A. K. 1992a, Phys. Rev. Lett., 68, 1488 (NP1)

2. 1992b, ApJ, 397, 729 (NP2)

$1992 \mathrm{c}$ Phys Re

1993, J. Phys. B, 26,1109

1994, Phys. Rev. A, 49, 1816 (NP3)

Nussbaumer, H., \& Storey, P.J. 1983, A\&A, 126, 75

- 1986, A\&AS, 64, 545 (NS)

Pradhan, A. K. \& Seaton, M.J. 1985, J. Phys. B, 18, 1631

Osterbrock, D. E. 1989, Astrophysics of Gaseous Nebulac and Active Galactic Nuclei (Mill Valley, CA: University Science Books)
Sakimoto, K., Terao, M., \& Berrington, K. A. 1990, Phys. Rev. A, 42, 291 Seaton, M. J. 1987, J. Phys. B, 20, 6363

Seaton, M. J., \& Storey, P. J. 1976, Atomic Processes and Applications, ed.

P. G. Burke \& B. L. Moiseiwitch (Amsterdam: North-Holland), 33

Shull, J. M., \& Steenberg, M. V. 1982, ApJ, 48, 95 (values are fitted from Jacobs et al. 1977)

Spitzer, L., Jr. \& Fitzpatrick, E. L. 1993, ApJ, 409, 299

Yu, Y., \& Seaton, M. J. 1987, J. Phys. B, 20, 6409 